Kansas State University Libraries

New Prairie Press

\title{
SUGGESTIONS FOR PRESENTING KRIGING RESULTS
}

D. W. Meek

T. J. Sauer

Follow this and additional works at: https://newprairiepress.org/agstatconference

Part of the Agriculture Commons, and the Applied Statistics Commons

\section{(c) $($ () $\ominus$}

This work is licensed under a Creative Commons Attribution-Noncommercial-No Derivative Works 4.0 License.

\section{Recommended Citation}

Meek, D. W. and Sauer, T. J. (2003). "SUGGESTIONS FOR PRESENTING KRIGING RESULTS," Conference on Applied Statistics in Agriculture. https://doi.org/10.4148/2475-7772.1191

This is brought to you for free and open access by the Conferences at New Prairie Press. It has been accepted for inclusion in Conference on Applied Statistics in Agriculture by an authorized administrator of New Prairie Press. For more information, please contact cads@k-state.edu. 


\title{
SUGGESTIONS FOR PRESENTING KRIGING RESULTS
}

\author{
D.W. Meek and T.J. Sauer \\ USDA-ARS-MWA-NSTL \\ Ames, IA 50011-4420 USA
}

\begin{abstract}
Kriging maps are often part of the reported analyses in many environmental research studies including those our agency is working on in the area of precision/sustainable farming. All to often important details on the underlying variography and/or kriging procedures are omitted. Likewise the content and form of presenting kriging results vary greatly. Often features of the underlying variability are not readily seen. Instead of reviewing poor practice in current literature, we offer guidelines for reporting the methodology and presenting the results with the use of soil test phosphorus (STP) measures from a real world pasture study. Relevantly, the stationarity assumption for the variogram is argued; computational aspects for both the model and empirical variogram development are reported; and similarly, computational aspects for the kriging surface are reported. In short, enough detail is reported to understand and reproduce the analyses. Standard practice for presenting kriging results should include both the kriging estimates and the associated standard error map. Various planar and three dimensional plots are shown and discussed. Emphasis is on developing quality gray-scale planar maps for conventional publications. Ideally, for both recommended plots, patterns and unique features of the surfaces' variability are revealed.
\end{abstract}

Keywords: angle tolerance, bandwidth, binning, data grid, kriging grid, kriging neighborhood, lag tolerance, the practical rule, the pragmatic compromise weight, marginal plots, isopleth maps, contour maps

\section{Introduction}

The analyses reported in many agricultural and environmental research articles frequently includes geostatistical results and presents an associated kriging map. For example, a recent unrestricted search of the words, "soil and kriging," in the AGRICOLA database produces 154 hits. Unfortunately, important procedural details are commonly omitted; consequently, an interested reader often cannot know enough to assess what was really done, let alone the soundness of the analysis. Moreover underlying assumptions, like stationarity are rarely assessed. Finally, the graphical displays of kriging surfaces vary greatly, as do the depictions of the surfaces features and variability.

In this paper, we offer guidelines for reporting the methodology and presenting the results Rather than review examples of poor practice in current literature, we offer our own example. With the use of soil test phosphorus (STP) measures from a real world pasture study (Sauer and Meek, 2003), we report on both the variography and kriging methodology in what we consider sufficient detail; then we show and discuss options for presenting the kriging surface. 


\section{Background on Our Data}

Land application of animal manure using site-specific techniques has potential to optimize nutrient recycling and minimize offsite environmental impacts. With this goal in mind, one objective of a recent work was to characterize the spatial variation of STP and some other related chemical properties (not reported here) in two pastures having contrasting grazing and poultry litter management (Sauer and Meek, 2003). In this work we consider the STP results from one site studied in 1999 (Cellar Ridge, AR). It was a lightly grazed, 6-ha tall fescue (Festuca arundinacea Schreb.) pasture with a history of limited poultry litter application (Fig. 1). Soil cores $(0-0.15 \mathrm{~m})$ were collected at 66 grid points with $30-\mathrm{m}$ spacing distances along both the north/south and east/west directions; and then they were chemically analyzed for STP (via a Mehlich 3 extract). No single resulting measurement exceeded the suggested permissible 150 $\mathrm{mg} / \mathrm{kg}$ maximum. Admittedly the data set is on the sparse side for geostatistical analysis but it is fairly typical of many agronomic field studies.

Poultry litter is a known source of phosphorus compounds and minerals that tend to increase STP over time. Applying poultry litter to pastures is common practice in the Cellar Ridge area. Hence a precision application strategy for poultry litter would start by estimating the initial spatial distribution and uncertainty of STP, i.e., a kriged surface with its standard error map.

\section{Variography Methods and Results}

\subsection{Stationarity}

Some form of stationarity, generally at least intrinsic stationarity, is often assumed without an argument in favor (for formal definitions, see e.g., pp. 16-17 in Chiles and Delfiner, 1999). Reasons for this omission vary from simple ignorance to avoidance of the philosophical issues and computational work involved when the stationarity assumption is a poor one. While working with nonstationary data may involve more complicated modeling and related computational choices, examining the stationarity assumption can be readily done. The assessment is primary! It is often said that one person's signal (trend, drift, etc.) is another person's noise. The goals and objectives of the analysis, however, should be set by the researcher. Are simplicity and insight important? Considering trend can result in simpler models with added understanding of a given data set's innate variability. For example, in Cressie's (1993) analysis of coal-ash data, the east-west variability can be modeled with just a linear trend (see section 3.4, pp. 151-170 in Cressie, 1993). Of course an obvious but arguably less desirable alternative is the stationary model with a spatially dependent residual.

Here the possible large-scale trend in STP was evaluated in several different ways including exploratory data analyses (EDA), hybrid graphical/analytical tests like marginal mean plots and various 2- and 3-dimensional surface plots (denoted 2D and 3D hereafter). Fig. 2 shows one possible raw data montage that can help depict a trend. Some other possible forms of marginal summaries include marginal medians, marginal means and medians, marginal box-plots, and variance standardized pocket plots (see e.g. pp. 44-45 in Cressie, 1993). Fig. 3 shows some other possible $2 \mathrm{D}$ and $3 \mathrm{D}$ plots. Further alternative forms for $2 \mathrm{D}$ plots include data postings and indicator maps (see e.g. pp. 40-46 in Isaaks and Srivastava, 1989) and lag scatter plots (see e.g. p. 
39 in Cressie, 1993). Many unique montages and/or combinations of any of these forms can be considered. Each has its own costs and benefits. In the 2D forms the number of and values for isopleths contours levels are arbitrary; intervals, quantiles, or selected values can be used. No one way is ideal for capturing all aspects of the variability. Considering the distribution of the data may aid greatly selecting suitable contour levels or isopleth intervals. Similar considerations should be used for 3D discrete data or continuous surface plots. Smooth surfaces can be developed in several different ways from simple linear interpolation (shown in the top right panel of Fig. 2.) to nonparametric regression methods like thin-plate splines and lowess. Ideally the data should be examined interactivly. Different tilt angles, rotation angles, aspects, etc. can provide insight.

Many simple analyses are possible. Here first and second order response surfaces can be developed as functions of the coordinates. Also the variability of residuals from a two-cycle median polish ( $\mathrm{mp}$ ) can be compared to that of the raw data. As always, of course, omnidirectional and directional empirical variograms (EV) and correlograms developed from the raw data should exhibit no undesirable properties such as apparent lack of nonnegative definiteness in the EVs and no trend contamination patterns in the correlograms.

All results for the Cellar Ridge set favor the stationarity assumption. None of the panels in Figs. 2 and 3 depicts a large scale simple trend. The simple response surfaces had poor determination and residual variances were almost that of the raw data. Also $\delta_{\mathrm{mp}} / \delta_{\text {raw }} \approx 0.9$. Moreover, the omnidirectional and directional empirical variograms (EV) and correlograms were satisfactory. In practice it is not necessary to do every possible test but if the EDA graphics like marginal medians are not clear, consider examining the assumption another way. Thus we recommend that stationarity be assessed and that the method of assessment be reported.

\subsection{Empirical and Model Variograms}

While explicit mention of the software used is not necessary, adequate description of the procedures used including the default or options used is essential for understanding what was done. For the sake of completeness we report that SAS ${ }^{\circledR}$ PROC VARIOGRAM was used (SAS ${ }^{\circledR}$, 1996). Please note that the mention of a trade-name does not imply an endorsement by our agency. Both isotropic and directional regular method of moments empirical variograms (not semivariograms) were developed and examined. Robust estimates were considered but not used (Hawkins and Cressie, 1984). Following Journel and Huijbregts (1978) only points in the spatial series for lag distances up to approximately one-half the domain length were considered. Here, lag tolerance is not an issue because of the square grid, and the default value was half the lag interval. For irregularly space data, the binning procedure, the lag distance classes, and lag distance definition need to be described.

Six directional variograms were estimated every $30^{\circ}$ from $0^{\circ}$ to $150^{\circ}$. Basically, just two will adequately tell the story: One along the eastings (east/west direction) and the other along the northings. Direction, angle tolerance, and bandwidth can, in general, influence the resulting EV or EVs. Comparative EVs developed from different sets of restrictions should be considered. Handily in the ARCVIEW ${ }^{\mathrm{TM}}$ geostatistics module the evaluation can be done interactively (ESRI, 1998). Here the angle tolerance was restricted to $45^{\circ}$, no bandwidth restrictions were 
used for binning because of the limited size of the overall domain the especially for the possible separation distances along the eastings (the maximum width is 7 points).

At least two models for each variogram were considered. In order to avoid the bias of ordinary least squares estimation and the effort and complexities of generalized least squares estimation, Cressie's (1993, p. 96-7) pragmatic compromise weight was used. For an example in SAS ${ }^{\circledR}$ code see Gotway (1991). For simplicity the model that best interpolated the empirical variogram was selected. No pseudo-cross-validation techniques were employed because only one model interpolated reasonably well. A pseudo-cross-validation is similar to a jackknife method. On a selected subgrid of the measures, observations are with-held one at a time, and each with-held value is estimated via ordinary kriging. Here, however, each model variogram is the original, i.e., model parameters are not re-estimated for each one-out subset. The selection is the model with the minimum prediction/measure difference sum of squares (like the PRESS statistic). The overall modeling procedures followed those presented in Meek $(2001,2002)$ both of which are compilations of ideas found in many well-known texts and other publications.

Variogram results favor considering a spherical directional model without a nugget along the eastings (Fig.4). There was no spatial variation along the northings. Eq. [1],

$$
\gamma_{E}\left(h_{E}\right)=760\left\{\begin{array}{lr}
\left(1.5\left(h_{E} / 58.7\right)-0.5\left(h_{E} / 58.7\right)^{3}\right), & 0<h_{E} \leq 58.7 \\
1, & h_{E}>58.7
\end{array}\right\}
$$

is the selected model variogram; where $h_{E}$ is the displacement in the east/west direction.

In brief, along with stating that the regular method of moments or specific robust procedure that was used, we recommend reporting the angle classes (or isotropy assumption with assessment criteria), angle tolerance, bandwidth, binning criteria, grid characteristics, lag distance, lag tolerance, and maximum number of lags considered for estimating the EV. For each selected model variogram, we recommend reporting the model form, parameter estimates, selection criteria, and weight. Anisotropic equations are implicit when the preceding angle information is given and so need not be explicitly written out.

\section{Ordinary Kriging Procedure and Results}

\subsection{Kriging Method}

Adequate description of the procedures used, including the default or option selections, is needed to understand what was done. Here ordinary kriging was used (specifically, SAS ${ }^{\circledR}$ PROC KRIGE2D was used [SAS $\left.\left.{ }^{\circledR}, 1996\right]\right)$. The kriging grid should be defined in adequate detail. Fig. 5 shows both the data grid and the interpolation grid that we used. Notice the kriging grid is 25 times denser (a point every $7.5 \mathrm{~m}$ ) and is confined to an interior area in order to avoid spatial extrapolation problems (i.e., each interpolation point is within some polygon formed by measured data points). Furthermore the kriging routine was set to use a minimum of four neighboring measured data values with radial distance restriction of values up to a distance of 90 $\mathrm{m}$. The previously defined directional variogram was employed to estimate both STP predictions (denoted STP) and their standard errors (denoted STP SE). 
In short, we recommend reporting all relevant details the kriging procedure. For ordinary kriging the interpolation grid, the model isotropic or directional variogram(s), and influence neighborhoods or selected interpolation criteria all need to be stated.

\subsection{Kriging Presentation}

Most often kriging results are shown with a contour map of the estimates; rarely is the

Most often kriging results are shown with a contour map of the estimates; rarely is the
associated variability (variance or standard error map) shown or summarized. Ideally both are
needed! All the 2D and 3D figures and display considerations previously discussed are relevant
to presenting the STP and STP SE surfaces. Again, no one is perfect, all having their own set of
favorable and unfavorable features. There is a lot of good literature available to get guidance on
presenting kriging data. In the spirit of Huff's famous book (1954), Monmonier (1996) deals
with analogous map issues. Tufte (1983, 1990, 1997) concentrates on graphical methods for
effective presentation of all sorts of data including but not limited to spatial data. Similarly
Cleveland (1993, 1994) focuses on general graphical methods but mainly for exploratory data
analysis purposes. As with the raw data, considering the distribution of the kriging estimates may
aid in selecting suitable contour levels or isopleth intervals for the best (honest) depiction.
Restricting the number of classification intervals is recommended (Tufte, 1983; Monmonier,
1996); too many can be confusing or at least difficult to discern. For nonelectronic publications,
interactive 3D graphical analysis in which view angle, tilt, etc. can be changed is not possible. In
fixed form flat-land, it is probably best to look at each surface in more than one way. A variation
on the latter would be to inlay some on a smaller scale within another, being careful not to make
the inlaid graphs too small to provide useful insight (this is the reason why, in general, we prefer
graphs with equal sized panels). If space is a problem, overlays of some compatible forms that
reinforce each other are possible. For example, Fig. 6 shows a possible final gray-scale graphic
form presentation for this analysis. In each panel, features of the univariate central tendency and
distribution are selected to choose the selected contours overlaid on the selected isopleth
background highlighting the mounds, valleys, saddles, troughs, etc. Notice the dominant
north/south features follow the slope of the land.

Most often kriging results are shown with a contour map of the estimates; rarely is the
associated variability (variance or standard error map) shown or summarized. Ideally both are
needed! All the 2D and 3D figures and display considerations previously discussed are relevant
to presenting the STP and STP SE surfaces. Again, no one is perfect, all having their own set of
favorable and unfavorable features. There is a lot of good literature available to get guidance on
presenting kriging data. In the spirit of Huff's famous book (1954), Monmonier (1996) deals
with analogous map issues. Tufte (1983, 1990, 1997) concentrates on graphical methods for
effective presentation of all sorts of data including but not limited to spatial data. Similarly
Cleveland (1993, 1994) focuses on general graphical methods but mainly for exploratory data
analysis purposes. As with the raw data, considering the distribution of the kriging estimates may
aid in selecting suitable contour levels or isopleth intervals for the best (honest) depiction.
Restricting the number of classification intervals is recommended (Tufte, 1983; Monmonier,
1996); too many can be confusing or at least difficult to discern. For nonelectronic publications,
interactive 3D graphical analysis in which view angle, tilt, etc. can be changed is not possible. In
fixed form flat-land, it is probably best to look at each surface in more than one way. A variation
on the latter would be to inlay some on a smaller scale within another, being careful not to make
the inlaid graphs too small to provide useful insight (this is the reason why, in general, we prefer
graphs with equal sized panels). If space is a problem, overlays of some compatible forms that
reinforce each other are possible. For example, Fig. 6 shows a possible final gray-scale graphic
form presentation for this analysis. In each panel, features of the univariate central tendency and
distribution are selected to choose the selected contours overlaid on the selected isopleth
background highlighting the mounds, valleys, saddles, troughs, etc. Notice the dominant
north/south features follow the slope of the land.

Most often kriging results are shown with a contour map of the estimates; rarely is the
associated variability (variance or standard error map) shown or summarized. Ideally both are
needed! All the 2D and 3D figures and display considerations previously discussed are relevant
to presenting the STP and STP SE surfaces. Again, no one is perfect, all having their own set of
favorable and unfavorable features. There is a lot of good literature available to get guidance on
presenting kriging data. In the spirit of Huff's famous book (1954), Monmonier (1996) deals
with analogous map issues. Tufte (1983, 1990, 1997) concentrates on graphical methods for
effective presentation of all sorts of data including but not limited to spatial data. Similarly
Cleveland (1993, 1994) focuses on general graphical methods but mainly for exploratory data
analysis purposes. As with the raw data, considering the distribution of the kriging estimates may
aid in selecting suitable contour levels or isopleth intervals for the best (honest) depiction.
Restricting the number of classification intervals is recommended (Tufte, 1983; Monmonier,
1996); too many can be confusing or at least difficult to discern. For nonelectronic publications,
interactive 3D graphical analysis in which view angle, tilt, etc. can be changed is not possible. In
fixed form flat-land, it is probably best to look at each surface in more than one way. A variation
on the latter would be to inlay some on a smaller scale within another, being careful not to make
the inlaid graphs too small to provide useful insight (this is the reason why, in general, we prefer
graphs with equal sized panels). If space is a problem, overlays of some compatible forms that
reinforce each other are possible. For example, Fig. 6 shows a possible final gray-scale graphic
form presentation for this analysis. In each panel, features of the univariate central tendency and
distribution are selected to choose the selected contours overlaid on the selected isopleth
background highlighting the mounds, valleys, saddles, troughs, etc. Notice the dominant
north/south features follow the slope of the land.

Most often kriging results are shown with a contour map of the estimates; rarely is the
associated variability (variance or standard error map) shown or summarized. Ideally both are
needed! All the 2D and 3D figures and display considerations previously discussed are relevant
to presenting the STP and STP SE surfaces. Again, no one is perfect, all having their own set of
favorable and unfavorable features. There is a lot of good literature available to get guidance on
presenting kriging data. In the spirit of Huff's famous book (1954), Monmonier (1996) deals
with analogous map issues. Tufte (1983, 1990, 1997) concentrates on graphical methods for
effective presentation of all sorts of data including but not limited to spatial data. Similarly
Cleveland (1993, 1994) focuses on general graphical methods but mainly for exploratory data
analysis purposes. As with the raw data, considering the distribution of the kriging estimates may
aid in selecting suitable contour levels or isopleth intervals for the best (honest) depiction.
Restricting the number of classification intervals is recommended (Tufte, 1983; Monmonier,
1996); too many can be confusing or at least difficult to discern. For nonelectronic publications,
interactive 3D graphical analysis in which view angle, tilt, etc. can be changed is not possible. In
fixed form flat-land, it is probably best to look at each surface in more than one way. A variation
on the latter would be to inlay some on a smaller scale within another, being careful not to make
the inlaid graphs too small to provide useful insight (this is the reason why, in general, we prefer
graphs with equal sized panels). If space is a problem, overlays of some compatible forms that
reinforce each other are possible. For example, Fig. 6 shows a possible final gray-scale graphic
form presentation for this analysis. In each panel, features of the univariate central tendency and
distribution are selected to choose the selected contours overlaid on the selected isopleth
background highlighting the mounds, valleys, saddles, troughs, etc. Notice the dominant
north/south features follow the slope of the land.

Most often kriging results are shown with a contour map of the estimates; rarely is the
associated variability (variance or standard error map) shown or summarized. Ideally both are
needed! All the 2D and 3D figures and display considerations previously discussed are relevant
to presenting the STP and STP SE surfaces. Again, no one is perfect, all having their own set of
favorable and unfavorable features. There is a lot of good literature available to get guidance on
presenting kriging data. In the spirit of Huff's famous book (1954), Monmonier (1996) deals
with analogous map issues. Tufte (1983, 1990, 1997) concentrates on graphical methods for
effective presentation of all sorts of data including but not limited to spatial data. Similarly
Cleveland (1993, 1994) focuses on general graphical methods but mainly for exploratory data
analysis purposes. As with the raw data, considering the distribution of the kriging estimates may
aid in selecting suitable contour levels or isopleth intervals for the best (honest) depiction.
Restricting the number of classification intervals is recommended (Tufte, 1983; Monmonier,
1996); too many can be confusing or at least difficult to discern. For nonelectronic publications,
interactive 3D graphical analysis in which view angle, tilt, etc. can be changed is not possible. In
fixed form flat-land, it is probably best to look at each surface in more than one way. A variation
on the latter would be to inlay some on a smaller scale within another, being careful not to make
the inlaid graphs too small to provide useful insight (this is the reason why, in general, we prefer
graphs with equal sized panels). If space is a problem, overlays of some compatible forms that
reinforce each other are possible. For example, Fig. 6 shows a possible final gray-scale graphic
form presentation for this analysis. In each panel, features of the univariate central tendency and
distribution are selected to choose the selected contours overlaid on the selected isopleth
background highlighting the mounds, valleys, saddles, troughs, etc. Notice the dominant
north/south features follow the slope of the land.

Most often kriging results are shown with a contour map of the estimates; rarely is the
associated variability (variance or standard error map) shown or summarized. Ideally both are
needed! All the 2D and 3D figures and display considerations previously discussed are relevant
to presenting the STP and STP SE surfaces. Again, no one is perfect, all having their own set of
favorable and unfavorable features. There is a lot of good literature available to get guidance on
presenting kriging data. In the spirit of Huff's famous book (1954), Monmonier (1996) deals
with analogous map issues. Tufte (1983, 1990, 1997) concentrates on graphical methods for
effective presentation of all sorts of data including but not limited to spatial data. Similarly
Cleveland (1993, 1994) focuses on general graphical methods but mainly for exploratory data
analysis purposes. As with the raw data, considering the distribution of the kriging estimates may
aid in selecting suitable contour levels or isopleth intervals for the best (honest) depiction.
Restricting the number of classification intervals is recommended (Tufte, 1983; Monmonier,
1996); too many can be confusing or at least difficult to discern. For nonelectronic publications,
interactive 3D graphical analysis in which view angle, tilt, etc. can be changed is not possible. In
fixed form flat-land, it is probably best to look at each surface in more than one way. A variation
on the latter would be to inlay some on a smaller scale within another, being careful not to make
the inlaid graphs too small to provide useful insight (this is the reason why, in general, we prefer
graphs with equal sized panels). If space is a problem, overlays of some compatible forms that
reinforce each other are possible. For example, Fig. 6 shows a possible final gray-scale graphic
form presentation for this analysis. In each panel, features of the univariate central tendency and
distribution are selected to choose the selected contours overlaid on the selected isopleth
background highlighting the mounds, valleys, saddles, troughs, etc. Notice the dominant
north/south features follow the slope of the land.

Most often kriging results are shown with a contour map of the estimates; rarely is the
associated variability (variance or standard error map) shown or summarized. Ideally both are
needed! All the 2D and 3D figures and display considerations previously discussed are relevant
to presenting the STP and STP SE surfaces. Again, no one is perfect, all having their own set of
favorable and unfavorable features. There is a lot of good literature available to get guidance on
presenting kriging data. In the spirit of Huff's famous book (1954), Monmonier (1996) deals
with analogous map issues. Tufte (1983, 1990, 1997) concentrates on graphical methods for
effective presentation of all sorts of data including but not limited to spatial data. Similarly
Cleveland (1993, 1994) focuses on general graphical methods but mainly for exploratory data
analysis purposes. As with the raw data, considering the distribution of the kriging estimates may
aid in selecting suitable contour levels or isopleth intervals for the best (honest) depiction.
Restricting the number of classification intervals is recommended (Tufte, 1983; Monmonier,
1996); too many can be confusing or at least difficult to discern. For nonelectronic publications,
interactive 3D graphical analysis in which view angle, tilt, etc. can be changed is not possible. In
fixed form flat-land, it is probably best to look at each surface in more than one way. A variation
on the latter would be to inlay some on a smaller scale within another, being careful not to make
the inlaid graphs too small to provide useful insight (this is the reason why, in general, we prefer
graphs with equal sized panels). If space is a problem, overlays of some compatible forms that
reinforce each other are possible. For example, Fig. 6 shows a possible final gray-scale graphic
form presentation for this analysis. In each panel, features of the univariate central tendency and
distribution are selected to choose the selected contours overlaid on the selected isopleth
background highlighting the mounds, valleys, saddles, troughs, etc. Notice the dominant
north/south features follow the slope of the land.

Most often kriging results are shown with a contour map of the estimates; rarely is the
associated variability (variance or standard error map) shown or summarized. Ideally both are
needed! All the 2D and 3D figures and display considerations previously discussed are relevant
to presenting the STP and STP SE surfaces. Again, no one is perfect, all having their own set of
favorable and unfavorable features. There is a lot of good literature available to get guidance on
presenting kriging data. In the spirit of Huff's famous book (1954), Monmonier (1996) deals
with analogous map issues. Tufte (1983, 1990, 1997) concentrates on graphical methods for
effective presentation of all sorts of data including but not limited to spatial data. Similarly
Cleveland (1993, 1994) focuses on general graphical methods but mainly for exploratory data
analysis purposes. As with the raw data, considering the distribution of the kriging estimates may
aid in selecting suitable contour levels or isopleth intervals for the best (honest) depiction.
Restricting the number of classification intervals is recommended (Tufte, 1983; Monmonier,
1996); too many can be confusing or at least difficult to discern. For nonelectronic publications,
interactive 3D graphical analysis in which view angle, tilt, etc. can be changed is not possible. In
fixed form flat-land, it is probably best to look at each surface in more than one way. A variation
on the latter would be to inlay some on a smaller scale within another, being careful not to make
the inlaid graphs too small to provide useful insight (this is the reason why, in general, we prefer
graphs with equal sized panels). If space is a problem, overlays of some compatible forms that
reinforce each other are possible. For example, Fig. 6 shows a possible final gray-scale graphic
form presentation for this analysis. In each panel, features of the univariate central tendency and
distribution are selected to choose the selected contours overlaid on the selected isopleth
background highlighting the mounds, valleys, saddles, troughs, etc. Notice the dominant
north/south features follow the slope of the land.

Most often kriging results are shown with a contour map of the estimates; rarely is the
associated variability (variance or standard error map) shown or summarized. Ideally both are
needed! All the 2D and 3D figures and display considerations previously discussed are relevant
to presenting the STP and STP SE surfaces. Again, no one is perfect, all having their own set of
favorable and unfavorable features. There is a lot of good literature available to get guidance on
presenting kriging data. In the spirit of Huff's famous book (1954), Monmonier (1996) deals
with analogous map issues. Tufte (1983, 1990, 1997) concentrates on graphical methods for
effective presentation of all sorts of data including but not limited to spatial data. Similarly
Cleveland (1993, 1994) focuses on general graphical methods but mainly for exploratory data
analysis purposes. As with the raw data, considering the distribution of the kriging estimates may
aid in selecting suitable contour levels or isopleth intervals for the best (honest) depiction.
Restricting the number of classification intervals is recommended (Tufte, 1983; Monmonier,
1996); too many can be confusing or at least difficult to discern. For nonelectronic publications,
interactive 3D graphical analysis in which view angle, tilt, etc. can be changed is not possible. In
fixed form flat-land, it is probably best to look at each surface in more than one way. A variation
on the latter would be to inlay some on a smaller scale within another, being careful not to make
the inlaid graphs too small to provide useful insight (this is the reason why, in general, we prefer
graphs with equal sized panels). If space is a problem, overlays of some compatible forms that
reinforce each other are possible. For example, Fig. 6 shows a possible final gray-scale graphic
form presentation for this analysis. In each panel, features of the univariate central tendency and
distribution are selected to choose the selected contours overlaid on the selected isopleth
background highlighting the mounds, valleys, saddles, troughs, etc. Notice the dominant
north/south features follow the slope of the land.

Most often kriging results are shown with a contour map of the estimates; rarely is the
associated variability (variance or standard error map) shown or summarized. Ideally both are
needed! All the 2D and 3D figures and display considerations previously discussed are relevant
to presenting the STP and STP SE surfaces. Again, no one is perfect, all having their own set of
favorable and unfavorable features. There is a lot of good literature available to get guidance on
presenting kriging data. In the spirit of Huff's famous book (1954), Monmonier (1996) deals
with analogous map issues. Tufte (1983, 1990, 1997) concentrates on graphical methods for
effective presentation of all sorts of data including but not limited to spatial data. Similarly
Cleveland (1993, 1994) focuses on general graphical methods but mainly for exploratory data
analysis purposes. As with the raw data, considering the distribution of the kriging estimates may
aid in selecting suitable contour levels or isopleth intervals for the best (honest) depiction.
Restricting the number of classification intervals is recommended (Tufte, 1983; Monmonier,
1996); too many can be confusing or at least difficult to discern. For nonelectronic publications,
interactive 3D graphical analysis in which view angle, tilt, etc. can be changed is not possible. In
fixed form flat-land, it is probably best to look at each surface in more than one way. A variation
on the latter would be to inlay some on a smaller scale within another, being careful not to make
the inlaid graphs too small to provide useful insight (this is the reason why, in general, we prefer
graphs with equal sized panels). If space is a problem, overlays of some compatible forms that
reinforce each other are possible. For example, Fig. 6 shows a possible final gray-scale graphic
form presentation for this analysis. In each panel, features of the univariate central tendency and
distribution are selected to choose the selected contours overlaid on the selected isopleth
background highlighting the mounds, valleys, saddles, troughs, etc. Notice the dominant
north/south features follow the slope of the land.

Most often kriging results are shown with a contour map of the estimates; rarely is the
associated variability (variance or standard error map) shown or summarized. Ideally both are
needed! All the 2D and 3D figures and display considerations previously discussed are relevant
to presenting the STP and STP SE surfaces. Again, no one is perfect, all having their own set of
favorable and unfavorable features. There is a lot of good literature available to get guidance on
presenting kriging data. In the spirit of Huff's famous book (1954), Monmonier (1996) deals
with analogous map issues. Tufte (1983, 1990, 1997) concentrates on graphical methods for
effective presentation of all sorts of data including but not limited to spatial data. Similarly
Cleveland (1993, 1994) focuses on general graphical methods but mainly for exploratory data
analysis purposes. As with the raw data, considering the distribution of the kriging estimates may
aid in selecting suitable contour levels or isopleth intervals for the best (honest) depiction.
Restricting the number of classification intervals is recommended (Tufte, 1983; Monmonier,
1996); too many can be confusing or at least difficult to discern. For nonelectronic publications,
interactive 3D graphical analysis in which view angle, tilt, etc. can be changed is not possible. In
fixed form flat-land, it is probably best to look at each surface in more than one way. A variation
on the latter would be to inlay some on a smaller scale within another, being careful not to make
the inlaid graphs too small to provide useful insight (this is the reason why, in general, we prefer
graphs with equal sized panels). If space is a problem, overlays of some compatible forms that
reinforce each other are possible. For example, Fig. 6 shows a possible final gray-scale graphic
form presentation for this analysis. In each panel, features of the univariate central tendency and
distribution are selected to choose the selected contours overlaid on the selected isopleth
background highlighting the mounds, valleys, saddles, troughs, etc. Notice the dominant
north/south features follow the slope of the land.

Most often kriging results are shown with a contour map of the estimates; rarely is the
associated variability (variance or standard error map) shown or summarized. Ideally both are
needed! All the 2D and 3D figures and display considerations previously discussed are relevant
to presenting the STP and STP SE surfaces. Again, no one is perfect, all having their own set of
favorable and unfavorable features. There is a lot of good literature available to get guidance on
presenting kriging data. In the spirit of Huff's famous book (1954), Monmonier (1996) deals
with analogous map issues. Tufte (1983, 1990, 1997) concentrates on graphical methods for
effective presentation of all sorts of data including but not limited to spatial data. Similarly
Cleveland (1993, 1994) focuses on general graphical methods but mainly for exploratory data
analysis purposes. As with the raw data, considering the distribution of the kriging estimates may
aid in selecting suitable contour levels or isopleth intervals for the best (honest) depiction.
Restricting the number of classification intervals is recommended (Tufte, 1983; Monmonier,
1996); too many can be confusing or at least difficult to discern. For nonelectronic publications,
interactive 3D graphical analysis in which view angle, tilt, etc. can be changed is not possible. In
fixed form flat-land, it is probably best to look at each surface in more than one way. A variation
on the latter would be to inlay some on a smaller scale within another, being careful not to make
the inlaid graphs too small to provide useful insight (this is the reason why, in general, we prefer
graphs with equal sized panels). If space is a problem, overlays of some compatible forms that
reinforce each other are possible. For example, Fig. 6 shows a possible final gray-scale graphic
form presentation for this analysis. In each panel, features of the univariate central tendency and
distribution are selected to choose the selected contours overlaid on the selected isopleth
background highlighting the mounds, valleys, saddles, troughs, etc. Notice the dominant
north/south features follow the slope of the land.

Most often kriging results are shown with a contour map of the estimates; rarely is the
associated variability (variance or standard error map) shown or summarized. Ideally both are
needed! All the 2D and 3D figures and display considerations previously discussed are relevant
to presenting the STP and STP SE surfaces. Again, no one is perfect, all having their own set of
favorable and unfavorable features. There is a lot of good literature available to get guidance on
presenting kriging data. In the spirit of Huff's famous book (1954), Monmonier (1996) deals
with analogous map issues. Tufte (1983, 1990, 1997) concentrates on graphical methods for
effective presentation of all sorts of data including but not limited to spatial data. Similarly
Cleveland (1993, 1994) focuses on general graphical methods but mainly for exploratory data
analysis purposes. As with the raw data, considering the distribution of the kriging estimates may
aid in selecting suitable contour levels or isopleth intervals for the best (honest) depiction.
Restricting the number of classification intervals is recommended (Tufte, 1983; Monmonier,
1996); too many can be confusing or at least difficult to discern. For nonelectronic publications,
interactive 3D graphical analysis in which view angle, tilt, etc. can be changed is not possible. In
fixed form flat-land, it is probably best to look at each surface in more than one way. A variation
on the latter would be to inlay some on a smaller scale within another, being careful not to make
the inlaid graphs too small to provide useful insight (this is the reason why, in general, we prefer
graphs with equal sized panels). If space is a problem, overlays of some compatible forms that
reinforce each other are possible. For example, Fig. 6 shows a possible final gray-scale graphic
form presentation for this analysis. In each panel, features of the univariate central tendency and
distribution are selected to choose the selected contours overlaid on the selected isopleth
background highlighting the mounds, valleys, saddles, troughs, etc. Notice the dominant
north/south features follow the slope of the land.

Most often kriging results are shown with a contour map of the estimates; rarely is the
associated variability (variance or standard error map) shown or summarized. Ideally both are
needed! All the 2D and 3D figures and display considerations previously discussed are relevant
to presenting the STP and STP SE surfaces. Again, no one is perfect, all having their own set of
favorable and unfavorable features. There is a lot of good literature available to get guidance on
presenting kriging data. In the spirit of Huff's famous book (1954), Monmonier (1996) deals
with analogous map issues. Tufte (1983, 1990, 1997) concentrates on graphical methods for
effective presentation of all sorts of data including but not limited to spatial data. Similarly
Cleveland (1993, 1994) focuses on general graphical methods but mainly for exploratory data
analysis purposes. As with the raw data, considering the distribution of the kriging estimates may
aid in selecting suitable contour levels or isopleth intervals for the best (honest) depiction.
Restricting the number of classification intervals is recommended (Tufte, 1983; Monmonier,
1996); too many can be confusing or at least difficult to discern. For nonelectronic publications,
interactive 3D graphical analysis in which view angle, tilt, etc. can be changed is not possible. In
fixed form flat-land, it is probably best to look at each surface in more than one way. A variation
on the latter would be to inlay some on a smaller scale within another, being careful not to make
the inlaid graphs too small to provide useful insight (this is the reason why, in general, we prefer
graphs with equal sized panels). If space is a problem, overlays of some compatible forms that
reinforce each other are possible. For example, Fig. 6 shows a possible final gray-scale graphic
form presentation for this analysis. In each panel, features of the univariate central tendency and
distribution are selected to choose the selected contours overlaid on the selected isopleth
background highlighting the mounds, valleys, saddles, troughs, etc. Notice the dominant
north/south features follow the slope of the land.

Most often kriging results are shown with a contour map of the estimates; rarely is the
associated variability (variance or standard error map) shown or summarized. Ideally both are
needed! All the 2D and 3D figures and display considerations previously discussed are relevant
to presenting the STP and STP SE surfaces. Again, no one is perfect, all having their own set of
favorable and unfavorable features. There is a lot of good literature available to get guidance on
presenting kriging data. In the spirit of Huff's famous book (1954), Monmonier (1996) deals
with analogous map issues. Tufte (1983, 1990, 1997) concentrates on graphical methods for
effective presentation of all sorts of data including but not limited to spatial data. Similarly
Cleveland (1993, 1994) focuses on general graphical methods but mainly for exploratory data
analysis purposes. As with the raw data, considering the distribution of the kriging estimates may
aid in selecting suitable contour levels or isopleth intervals for the best (honest) depiction.
Restricting the number of classification intervals is recommended (Tufte, 1983; Monmonier,
1996); too many can be confusing or at least difficult to discern. For nonelectronic publications,
interactive 3D graphical analysis in which view angle, tilt, etc. can be changed is not possible. In
fixed form flat-land, it is probably best to look at each surface in more than one way. A variation
on the latter would be to inlay some on a smaller scale within another, being careful not to make
the inlaid graphs too small to provide useful insight (this is the reason why, in general, we prefer
graphs with equal sized panels). If space is a problem, overlays of some compatible forms that
reinforce each other are possible. For example, Fig. 6 shows a possible final gray-scale graphic
form presentation for this analysis. In each panel, features of the univariate central tendency and
distribution are selected to choose the selected contours overlaid on the selected isopleth
background highlighting the mounds, valleys, saddles, troughs, etc. Notice the dominant
north/south features follow the slope of the land.

Most often kriging results are shown with a contour map of the estimates; rarely is the
associated variability (variance or standard error map) shown or summarized. Ideally both are
needed! All the 2D and 3D figures and display considerations previously discussed are relevant
to presenting the STP and STP SE surfaces. Again, no one is perfect, all having their own set of
favorable and unfavorable features. There is a lot of good literature available to get guidance on
presenting kriging data. In the spirit of Huff's famous book (1954), Monmonier (1996) deals
with analogous map issues. Tufte (1983, 1990, 1997) concentrates on graphical methods for
effective presentation of all sorts of data including but not limited to spatial data. Similarly
Cleveland (1993, 1994) focuses on general graphical methods but mainly for exploratory data
analysis purposes. As with the raw data, considering the distribution of the kriging estimates may
aid in selecting suitable contour levels or isopleth intervals for the best (honest) depiction.
Restricting the number of classification intervals is recommended (Tufte, 1983; Monmonier,
1996); too many can be confusing or at least difficult to discern. For nonelectronic publications,
interactive 3D graphical analysis in which view angle, tilt, etc. can be changed is not possible. In
fixed form flat-land, it is probably best to look at each surface in more than one way. A variation
on the latter would be to inlay some on a smaller scale within another, being careful not to make
the inlaid graphs too small to provide useful insight (this is the reason why, in general, we prefer
graphs with equal sized panels). If space is a problem, overlays of some compatible forms that
reinforce each other are possible. For example, Fig. 6 shows a possible final gray-scale graphic
form presentation for this analysis. In each panel, features of the univariate central tendency and
distribution are selected to choose the selected contours overlaid on the selected isopleth
background highlighting the mounds, valleys, saddles, troughs, etc. Notice the dominant
north/south features follow the slope of the land.

Most often kriging results are shown with a contour map of the estimates; rarely is the
associated variability (variance or standard error map) shown or summarized. Ideally both are
needed! All the 2D and 3D figures and display considerations previously discussed are relevant
to presenting the STP and STP SE surfaces. Again, no one is perfect, all having their own set of
favorable and unfavorable features. There is a lot of good literature available to get guidance on
presenting kriging data. In the spirit of Huff's famous book (1954), Monmonier (1996) deals
with analogous map issues. Tufte (1983, 1990, 1997) concentrates on graphical methods for
effective presentation of all sorts of data including but not limited to spatial data. Similarly
Cleveland (1993, 1994) focuses on general graphical methods but mainly for exploratory data
analysis purposes. As with the raw data, considering the distribution of the kriging estimates may
aid in selecting suitable contour levels or isopleth intervals for the best (honest) depiction.
Restricting the number of classification intervals is recommended (Tufte, 1983; Monmonier,
1996); too many can be confusing or at least difficult to discern. For nonelectronic publications,
interactive 3D graphical analysis in which view angle, tilt, etc. can be changed is not possible. In
fixed form flat-land, it is probably best to look at each surface in more than one way. A variation
on the latter would be to inlay some on a smaller scale within another, being careful not to make
the inlaid graphs too small to provide useful insight (this is the reason why, in general, we prefer
graphs with equal sized panels). If space is a problem, overlays of some compatible forms that
reinforce each other are possible. For example, Fig. 6 shows a possible final gray-scale graphic
form presentation for this analysis. In each panel, features of the univariate central tendency and
distribution are selected to choose the selected contours overlaid on the selected isopleth
background highlighting the mounds, valleys, saddles, troughs, etc. Notice the dominant
north/south features follow the slope of the land.

Most often kriging results are shown with a contour map of the estimates; rarely is the
associated variability (variance or standard error map) shown or summarized. Ideally both are
needed! All the 2D and 3D figures and display considerations previously discussed are relevant
to presenting the STP and STP SE surfaces. Again, no one is perfect, all having their own set of
favorable and unfavorable features. There is a lot of good literature available to get guidance on
presenting kriging data. In the spirit of Huff's famous book (1954), Monmonier (1996) deals
with analogous map issues. Tufte (1983, 1990, 1997) concentrates on graphical methods for
effective presentation of all sorts of data including but not limited to spatial data. Similarly
Cleveland (1993, 1994) focuses on general graphical methods but mainly for exploratory data
analysis purposes. As with the raw data, considering the distribution of the kriging estimates may
aid in selecting suitable contour levels or isopleth intervals for the best (honest) depiction.
Restricting the number of classification intervals is recommended (Tufte, 1983; Monmonier,
1996); too many can be confusing or at least difficult to discern. For nonelectronic publications,
interactive 3D graphical analysis in which view angle, tilt, etc. can be changed is not possible. In
fixed form flat-land, it is probably best to look at each surface in more than one way. A variation
on the latter would be to inlay some on a smaller scale within another, being careful not to make
the inlaid graphs too small to provide useful insight (this is the reason why, in general, we prefer
graphs with equal sized panels). If space is a problem, overlays of some compatible forms that
reinforce each other are possible. For example, Fig. 6 shows a possible final gray-scale graphic
form presentation for this analysis. In each panel, features of the univariate central tendency and
distribution are selected to choose the selected contours overlaid on the selected isopleth
background highlighting the mounds, valleys, saddles, troughs, etc. Notice the dominant
north/south features follow the slope of the land.

Most often kriging results are shown with a contour map of the estimates; rarely is the
associated variability (variance or standard error map) shown or summarized. Ideally both are
needed! All the 2D and 3D figures and display considerations previously discussed are relevant
to presenting the STP and STP SE surfaces. Again, no one is perfect, all having their own set of
favorable and unfavorable features. There is a lot of good literature available to get guidance on
presenting kriging data. In the spirit of Huff's famous book (1954), Monmonier (1996) deals
with analogous map issues. Tufte (1983, 1990, 1997) concentrates on graphical methods for
effective presentation of all sorts of data including but not limited to spatial data. Similarly
Cleveland (1993, 1994) focuses on general graphical methods but mainly for exploratory data
analysis purposes. As with the raw data, considering the distribution of the kriging estimates may
aid in selecting suitable contour levels or isopleth intervals for the best (honest) depiction.
Restricting the number of classification intervals is recommended (Tufte, 1983; Monmonier,
1996); too many can be confusing or at least difficult to discern. For nonelectronic publications,
interactive 3D graphical analysis in which view angle, tilt, etc. can be changed is not possible. In
fixed form flat-land, it is probably best to look at each surface in more than one way. A variation
on the latter would be to inlay some on a smaller scale within another, being careful not to make
the inlaid graphs too small to provide useful insight (this is the reason why, in general, we prefer
graphs with equal sized panels). If space is a problem, overlays of some compatible forms that
reinforce each other are possible. For example, Fig. 6 shows a possible final gray-scale graphic
form presentation for this analysis. In each panel, features of the univariate central tendency and
distribution are selected to choose the selected contours overlaid on the selected isopleth
background highlighting the mounds, valleys, saddles, troughs, etc. Notice the dominant
north/south features follow the slope of the land.

Most often kriging results are shown with a contour map of the estimates; rarely is the
associated variability (variance or standard error map) shown or summarized. Ideally both are
needed! All the 2D and 3D figures and display considerations previously discussed are relevant
to presenting the STP and STP SE surfaces. Again, no one is perfect, all having their own set of
favorable and unfavorable features. There is a lot of good literature available to get guidance on
presenting kriging data. In the spirit of Huff's famous book (1954), Monmonier (1996) deals
with analogous map issues. Tufte (1983, 1990, 1997) concentrates on graphical methods for
effective presentation of all sorts of data including but not limited to spatial data. Similarly
Cleveland (1993, 1994) focuses on general graphical methods but mainly for exploratory data
analysis purposes. As with the raw data, considering the distribution of the kriging estimates may
aid in selecting suitable contour levels or isopleth intervals for the best (honest) depiction.
Restricting the number of classification intervals is recommended (Tufte, 1983; Monmonier,
1996); too many can be confusing or at least difficult to discern. For nonelectronic publications,
interactive 3D graphical analysis in which view angle, tilt, etc. can be changed is not possible. In
fixed form flat-land, it is probably best to look at each surface in more than one way. A variation
on the latter would be to inlay some on a smaller scale within another, being careful not to make
the inlaid graphs too small to provide useful insight (this is the reason why, in general, we prefer
graphs with equal sized panels). If space is a problem, overlays of some compatible forms that
reinforce each other are possible. For example, Fig. 6 shows a possible final gray-scale graphic
form presentation for this analysis. In each panel, features of the univariate central tendency and
distribution are selected to choose the selected contours overlaid on the selected isopleth
background highlighting the mounds, valleys, saddles, troughs, etc. Notice the dominant
north/south features follow the slope of the land.

Most often kriging results are shown with a contour map of the estimates; rarely is the
associated variability (variance or standard error map) shown or summarized. Ideally both are
needed! All the 2D and 3D figures and display considerations previously discussed are relevant
to presenting the STP and STP SE surfaces. Again, no one is perfect, all having their own set of
favorable and unfavorable features. There is a lot of good literature available to get guidance on
presenting kriging data. In the spirit of Huff's famous book (1954), Monmonier (1996) deals
with analogous map issues. Tufte (1983, 1990, 1997) concentrates on graphical methods for
effective presentation of all sorts of data including but not limited to spatial data. Similarly
Cleveland (1993, 1994) focuses on general graphical methods but mainly for exploratory data
analysis purposes. As with the raw data, considering the distribution of the kriging estimates may
aid in selecting suitable contour levels or isopleth intervals for the best (honest) depiction.
Restricting the number of classification intervals is recommended (Tufte, 1983; Monmonier,
1996); too many can be confusing or at least difficult to discern. For nonelectronic publications,
interactive 3D graphical analysis in which view angle, tilt, etc. can be changed is not possible. In
fixed form flat-land, it is probably best to look at each surface in more than one way. A variation
on the latter would be to inlay some on a smaller scale within another, being careful not to make
the inlaid graphs too small to provide useful insight (this is the reason why, in general, we prefer
graphs with equal sized panels). If space is a problem, overlays of some compatible forms that
reinforce each other are possible. For example, Fig. 6 shows a possible final gray-scale graphic
form presentation for this analysis. In each panel, features of the univariate central tendency and
distribution are selected to choose the selected contours overlaid on the selected isopleth
background highlighting the mounds, valleys, saddles, troughs, etc. Notice the dominant
north/south features follow the slope of the land.

Most often kriging results are shown with a contour map of the estimates; rarely is the
associated variability (variance or standard error map) shown or summarized. Ideally both are
needed! All the 2D and 3D figures and display considerations previously discussed are relevant
to presenting the STP and STP SE surfaces. Again, no one is perfect, all having their own set of
favorable and unfavorable features. There is a lot of good literature available to get guidance on
presenting kriging data. In the spirit of Huff's famous book (1954), Monmonier (1996) deals
with analogous map issues. Tufte (1983, 1990, 1997) concentrates on graphical methods for
effective presentation of all sorts of data including but not limited to spatial data. Similarly
Cleveland (1993, 1994) focuses on general graphical methods but mainly for exploratory data
analysis purposes. As with the raw data, considering the distribution of the kriging estimates may
aid in selecting suitable contour levels or isopleth intervals for the best (honest) depiction.
Restricting the number of classification intervals is recommended (Tufte, 1983; Monmonier,
1996); too many can be confusing or at least difficult to discern. For nonelectronic publications,
interactive 3D graphical analysis in which view angle, tilt, etc. can be changed is not possible. In
fixed form flat-land, it is probably best to look at each surface in more than one way. A variation
on the latter would be to inlay some on a smaller scale within another, being careful not to make
the inlaid graphs too small to provide useful insight (this is the reason why, in general, we prefer
graphs with equal sized panels). If space is a problem, overlays of some compatible forms that
reinforce each other are possible. For example, Fig. 6 shows a possible final gray-scale graphic
form presentation for this analysis. In each panel, features of the univariate central tendency and
distribution are selected to choose the selected contours overlaid on the selected isopleth
background highlighting the mounds, valleys, saddles, troughs, etc. Notice the dominant
north/south features follow the slope of the land.

\section{Summary}

Some assessment of the underlying stationarity and isotropy assumptions is vital. The reporting of detail on the software defaults or options employed is requisite for the EV, for the variogram model, and for the kriging procedure including the interpolation domain. Moreover, a well chosen display can capture the essence of the analysis and provide insight to the given application.

\section{Acknowledgments}

This work was supported by the USDA-ARS-MWA National Soil Tilth Laboratory, Ames, IA., Dr. J.L. Hatfield, Director. Prof. P. Dixon and Dr. R. Ewing, Iowa State University (ISU), Ames, IA., provided comments on the manuscript. Also thanks to one anonymous reviewer. The 
information in this work is a compilation of ideology found in most of the referenced works and from unpublished course notes by Prof. M. Kaiser for Statistics 406/506 taught at ISU in the Spring of 2002. The idea for the gray-scale overlays, however, is the first author's.

\section{References}

Chilès, J.P., and Delfiner, P. 1999. Geostatistics: Modeling Spatial Uncertainty. J. Wiley and Sons, Inc., New York, NY. 695 pp.

Cleveland, W.S. 1994. The Elements of Graphing Data. Hobart Press, Summit, NJ. 360 pp.

Cleveland, W.S. 1993. Visualizing Data. Hobart Press, Summit, NJ. 297 pp.

Cressie, N.A.C., 1993. Statistics for spatial data. J. Wiley \& Sons, New York, NY. 900 pp.

ESRI. 1998. Getting to know ARCVIEW GIS v. 3.1, ESRI Press,Redlands, CA. 660 pp.

Gotway, C.A. 1991. Fitting semivariogram models by weighted least squares. Computers \& Geosciences 17, 171-172.

Hawkins, D.M., and Cressie, N.A.C. 1984. Robust Kriging - A Proposal. J. Intl. Assoc. Math. Geol. 16: 3-18.

Huff, D. 1954. How to lie with statistics. W.W. Norton \& Co., New York, NY. 142 pp.

Isaaks, E.H. and Srivastava, R.M. 1989. An introduction to applied geostatistics. Oxford Univ. Press, New York, 561 pp.

Journel, A.G., and Huijbregts, Ch.J. 1978. Mining Geostatistics. Academic Press, New York, NY. 600 pp.

Meek, D.W., 2002. Another look at Clark's adit silver series, p.356-368. In: G. Milliken (ed.), Proc. 13 ${ }^{\text {th }}$ Appl. Stat. Agric. Conf., Manhattan, KS, Apr. 29 - May 1, 2001. Stat. Dept., KS. St. Univ., Manhattan, KS.

Meek, D.W. 2001. A semiparametric method for estimating the scale of fluctuation. Comp. \& Geosci. 27(10): 1243-1249.

Monmonier, M. 1996. How to lie with maps, $2^{\text {od }}$ Ed. Univ. Chicago Press, Chicago, Il. 207 pp.

$\mathrm{SAS}^{\circledR,}$ Institute. 1996. SAS/STAT ${ }^{\circledR,}$ technical report: Spatial prediciton using the $\mathrm{SAS}^{\circledR,}$ system, \#55715. SAS ${ }^{\circledR}$ Institute, Cary, NC, 72 pp. 


\section{Kansas State University}

Sauer, T.J. and D.W. Meek. 2003. Spatial variation of soil phosphorus in pastures with contrasting management. Soil Sci. Soc. Am. J.67: 826-836.

Tufte, E.R. 1983. The visual display of quantitative information. Graphics Press, Cheshire, CT. $197 \mathrm{pp}$.

Tufte, E.R. 1990. Envisioning Information. Graphics Press, Cheshire, CT. 126 pp.

Tufte, E.R. 1997. Visual Explanations. Graphics Press, Cheshire, CT. 156 pp. 


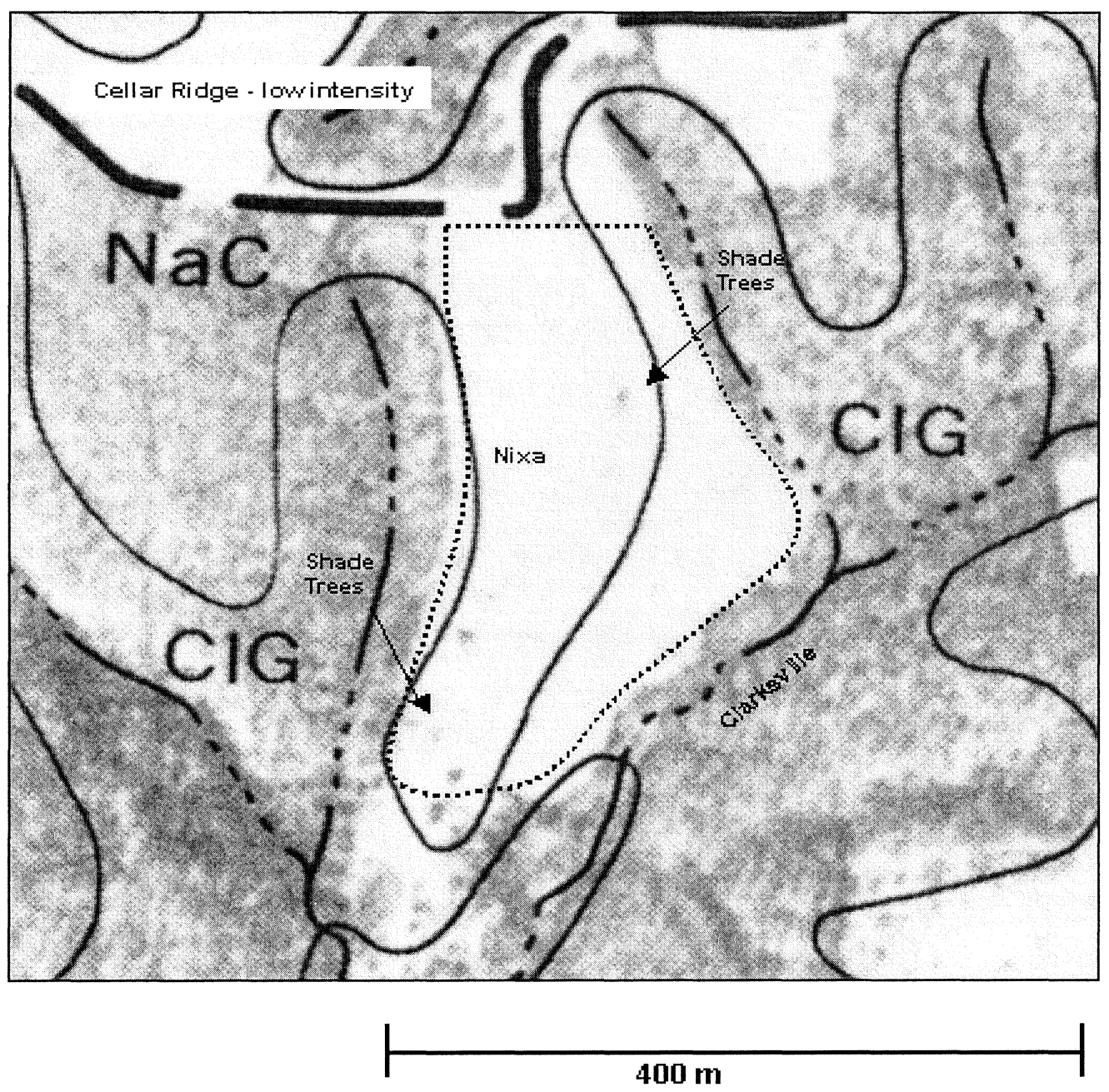

Figure 1. Soil map of the Cellar Ridge field with Nixa and Clarksville soils. The poultry litter management was low intensity. Dashed lines indicate the boundaries of the paddock sampled on a 30-m grid. The land has a general north-south direction slope with the higher ground to the north. The gray blurry spots are shade trees. 


\section{FOUR PERSPECTIVES}

NORTHING MARGINAL SUMMARIES

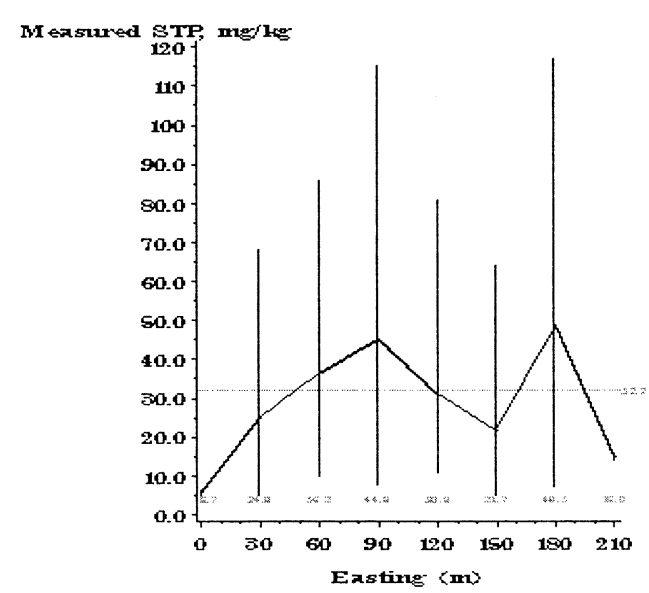

CONTOURS FOR SELECTED STP QUANTILES

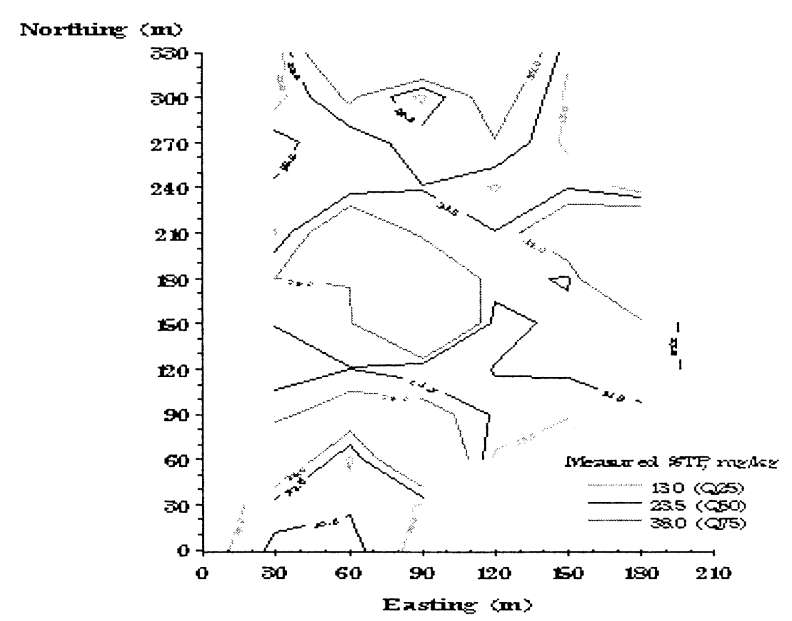

SIMPLE SURFACE PLOT Tilt $=75$, Rotate $=45$

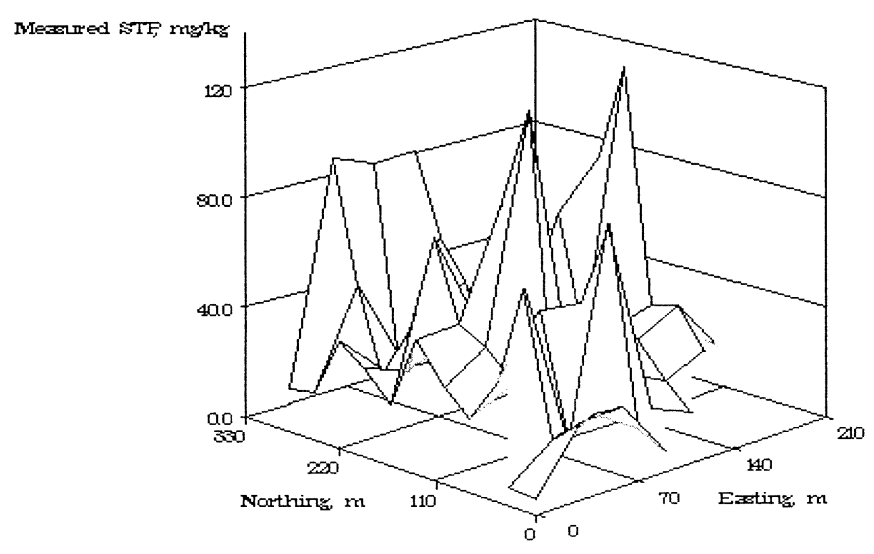

EASTING MARGINAL SUMMARIES

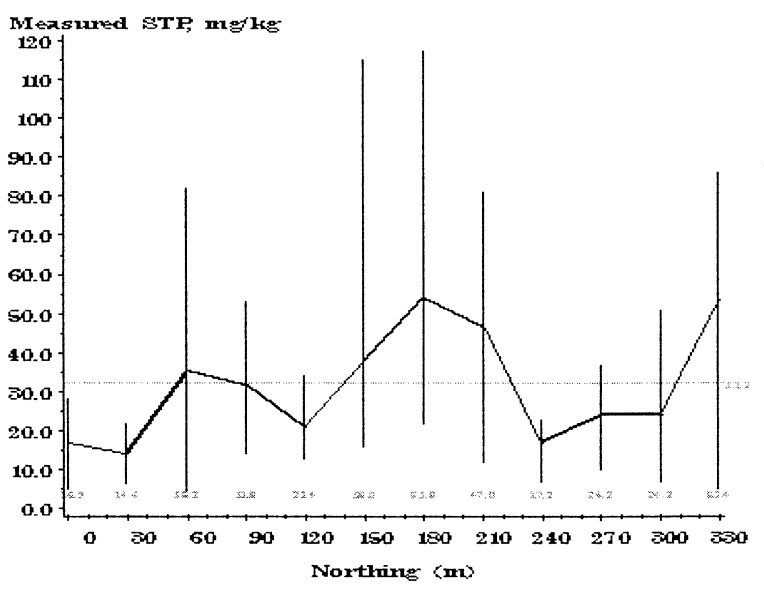

Figure 2. Draftsman like four panel montage of the raw data surface for the 1999 Cellar Ridge, AR STP samples (mg/kg). 


\section{SOME OTHER 2D AND 3D VIEWS}

QUANTILE SYMBOL MAP

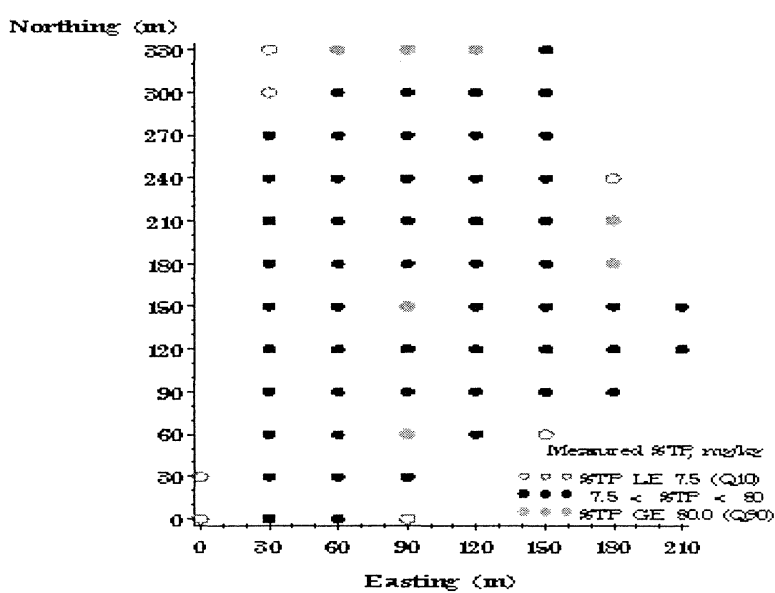

QUANTTLE ISOPLETH MAP

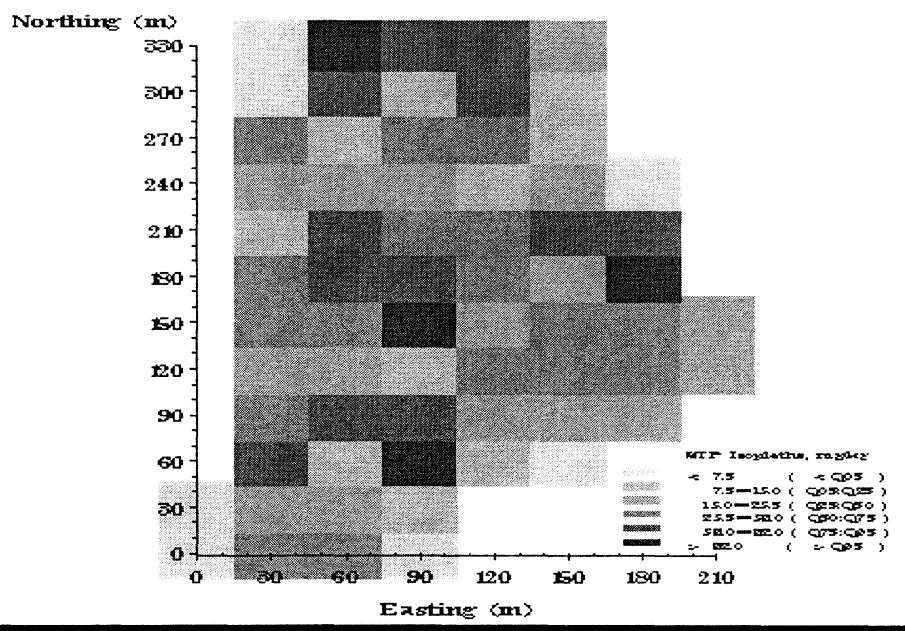

3D SCATIER PLOT, Tilt $=75$, Fotate $=45$

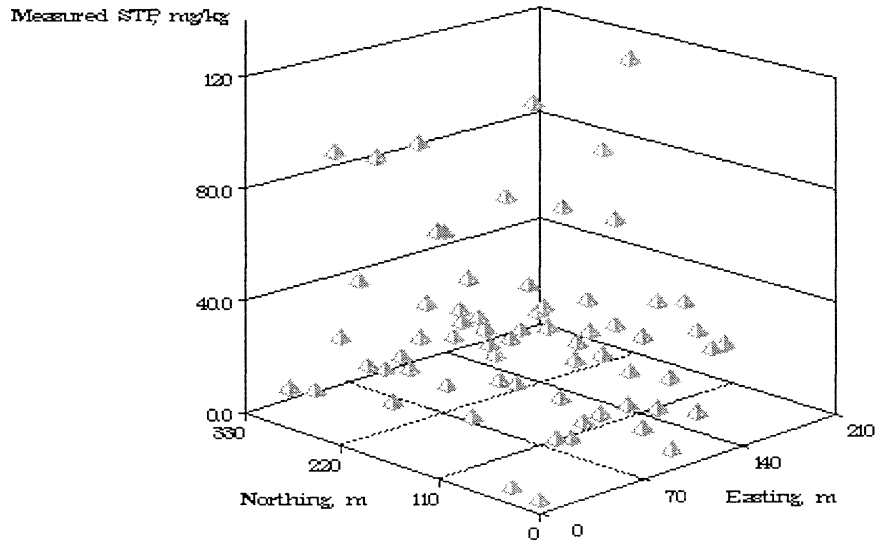

3D NEEDLE PLOT, TiH $=75$, Rotate $=45$

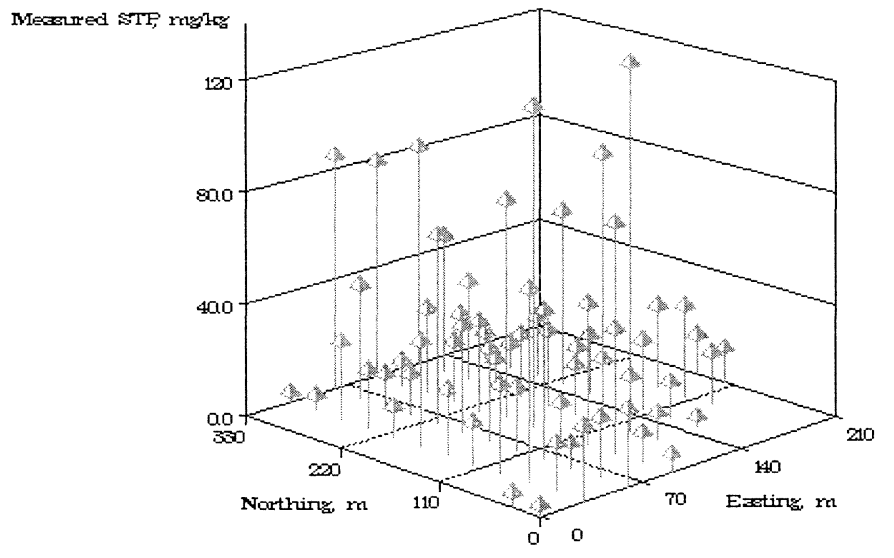

Figure 3. Alternative 2 and 3 dimensional graphs of the 1999 Cellar Ridge, AR STP $(\mathrm{mg} / \mathrm{kg})$ raw data samples. 


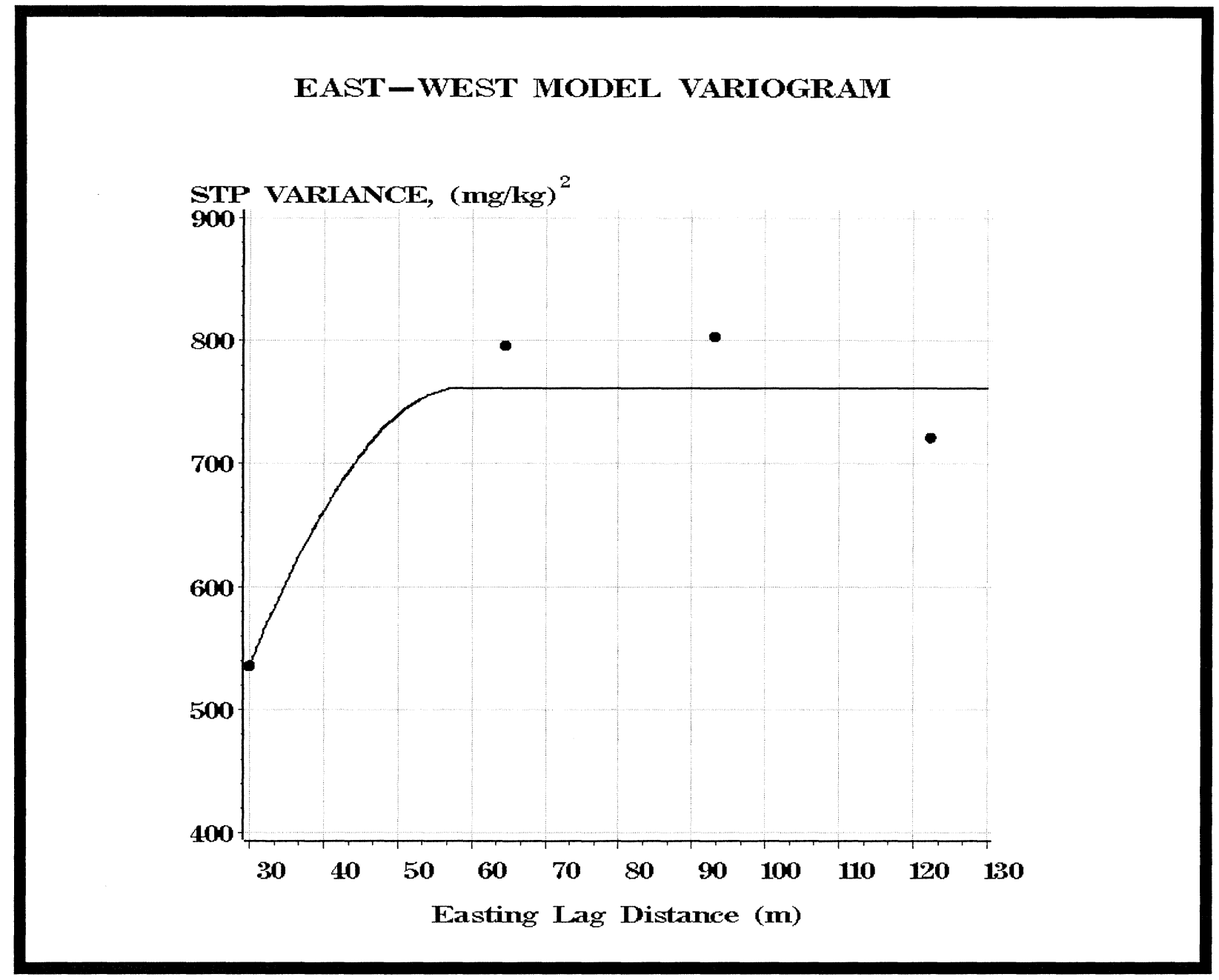

Figure 4. Directional variogram for the stationary STP data (in $\mathrm{mg} / \mathrm{kg}$ ) from Cellar Ridge, AR. 


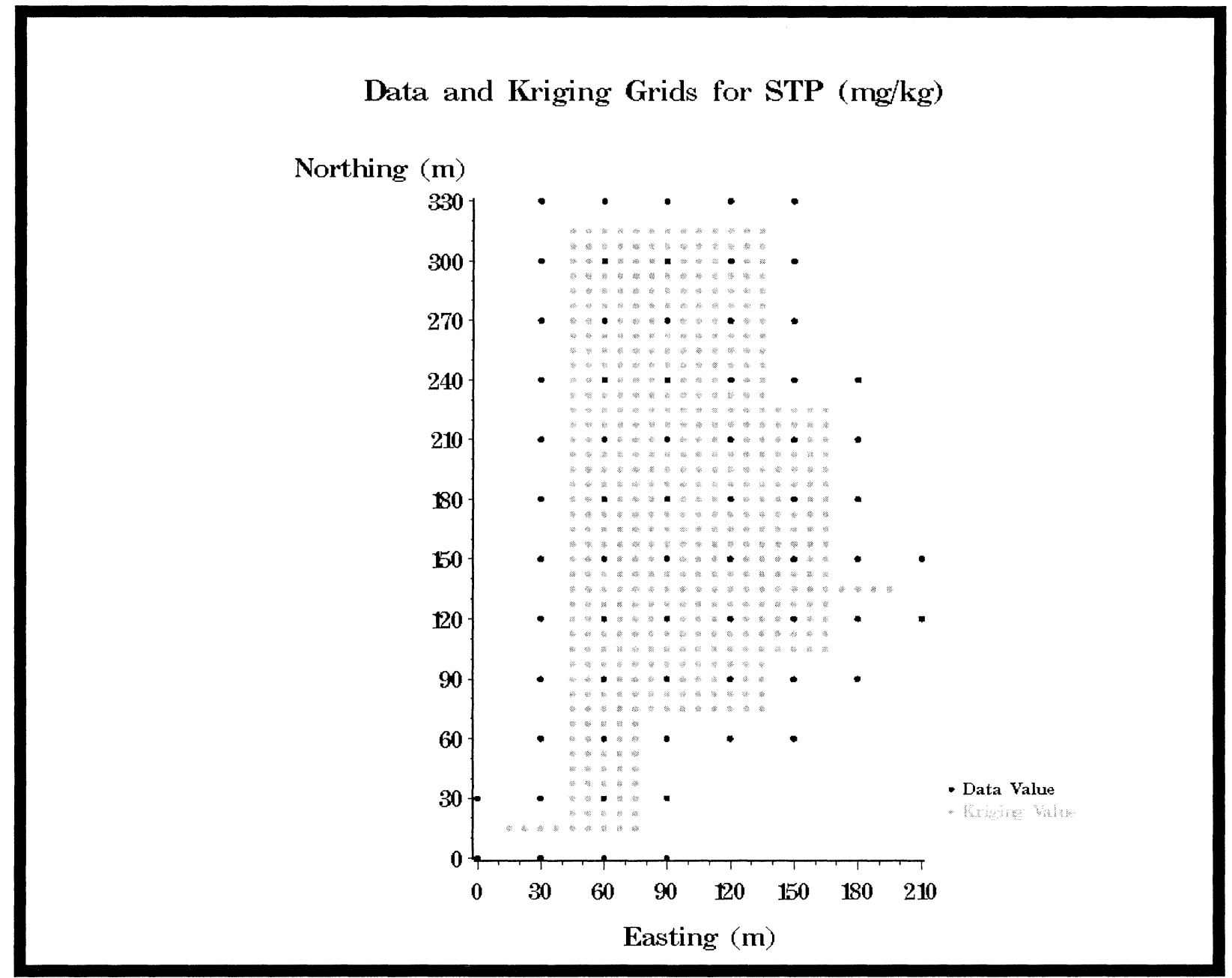

Figure 5. Data and kriging grids for the 1999 STP $(\mathrm{mg} / \mathrm{kg})$ study at Cellar Ridge, AR. Note the kriging grid is entirely inside the perimeter of the sampling grid so at least 4 surrounding points can be used for the kriging interpolation procedure. 


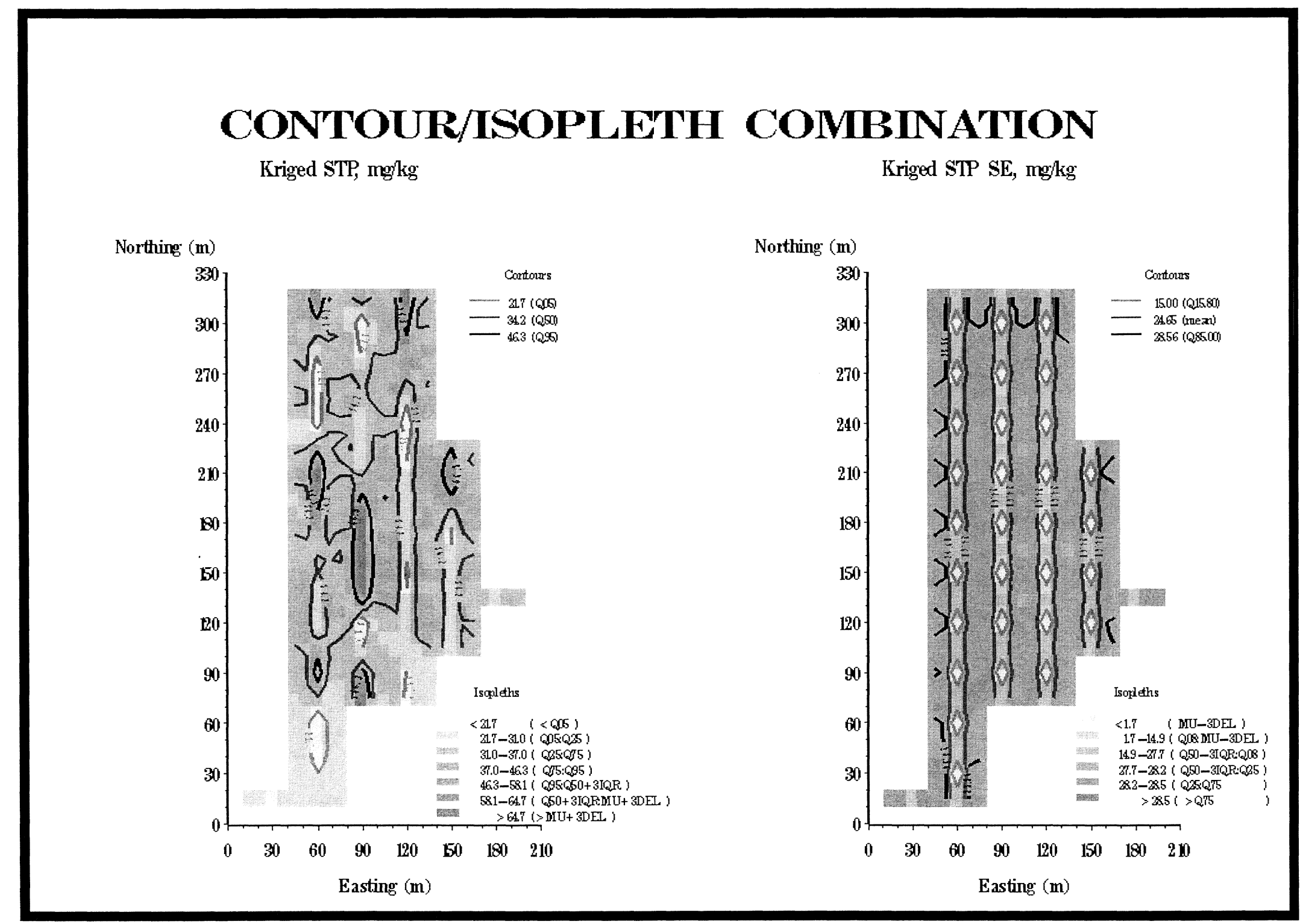

Figure 6. Suggested gray-scale contour/isopleth overlays for Cellar Ridge, AR kriged STP surface and its standard error $(\mathrm{mg} / \mathrm{kg})$ constructed from the 1999 samples. 


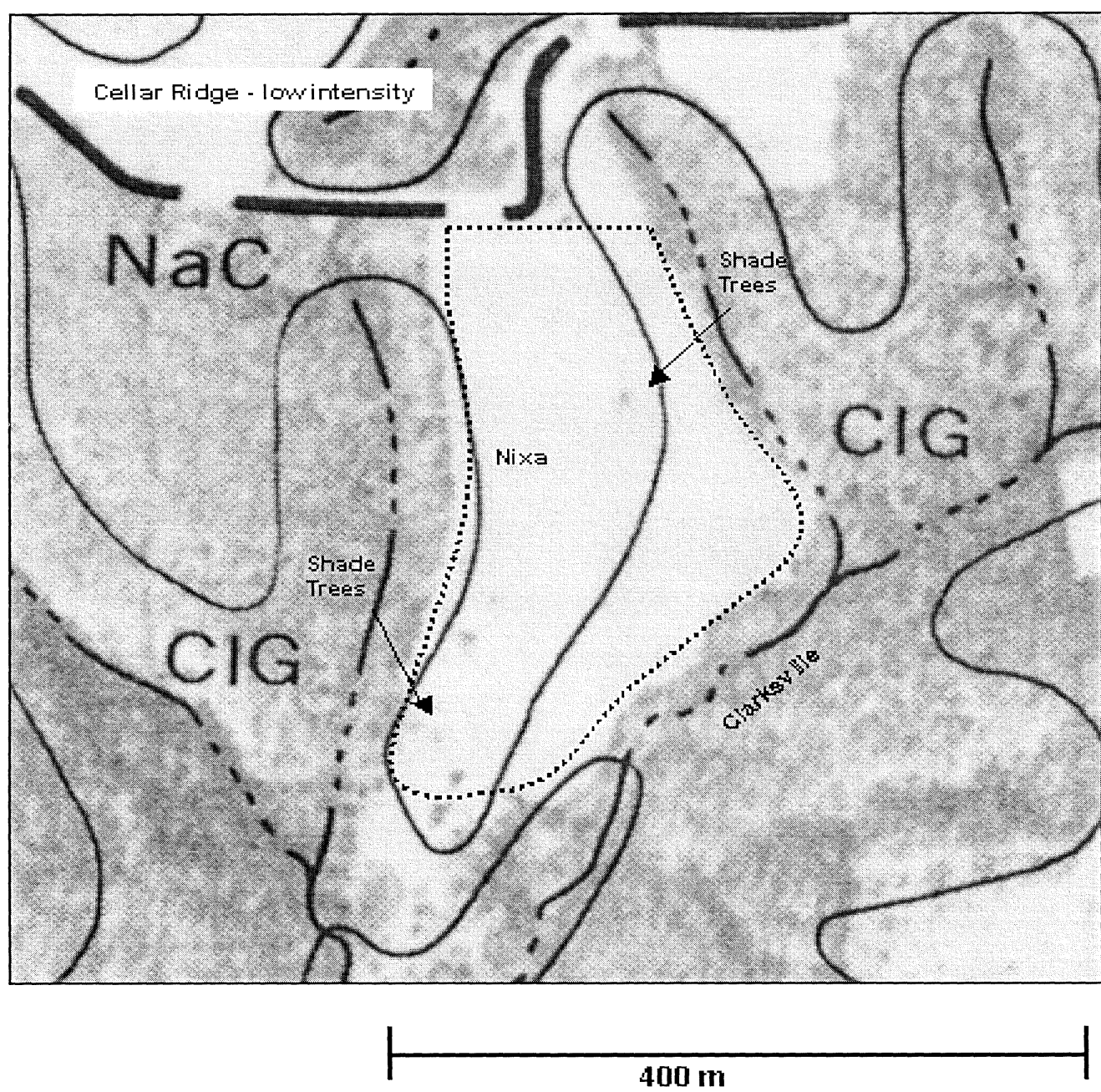

Figure 1. Soil map of the Cellar Ridge field with Nixa and Clarksville soils. The poultry litter management was low intensity. Dashed lines indicate the boundaries of the paddock sampled on a 30-m grid. The land has a general north-south direction slope with the higher ground to the north. The gray blurry spots are shade trees. 


\section{FOUR PERSPECTIVES}

NORTHING MAFGINAL SUMMARIES

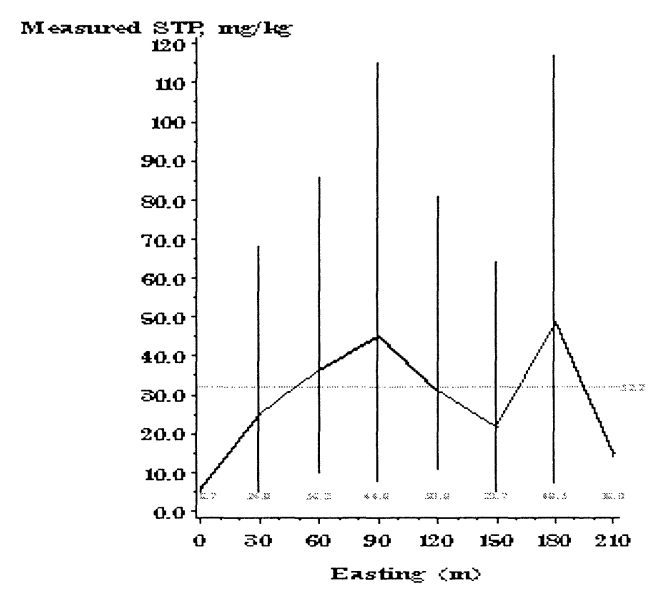

CONTOURS FOR SELECTED STP QUANTILES

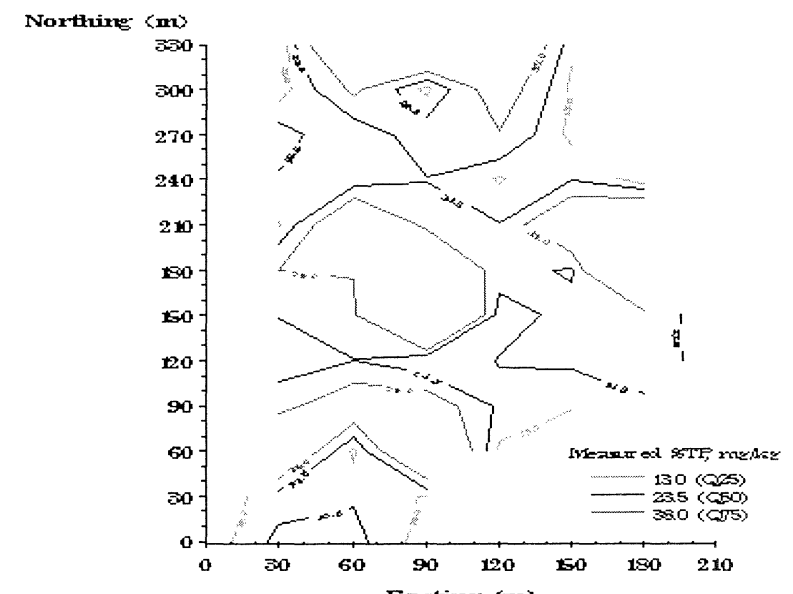

STMPLE SURFACE PLOT Tit $=75$, Rotate $=45$

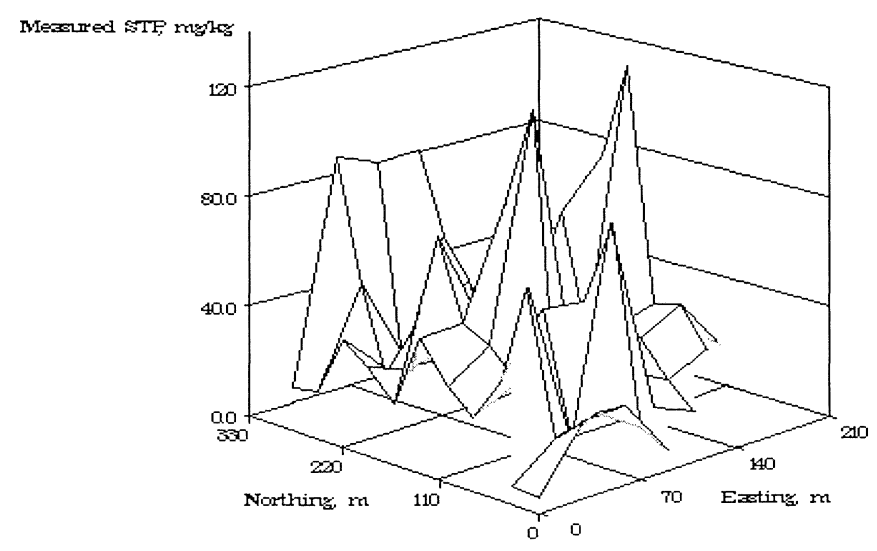

EASTING MARGINAL SUMMARIES

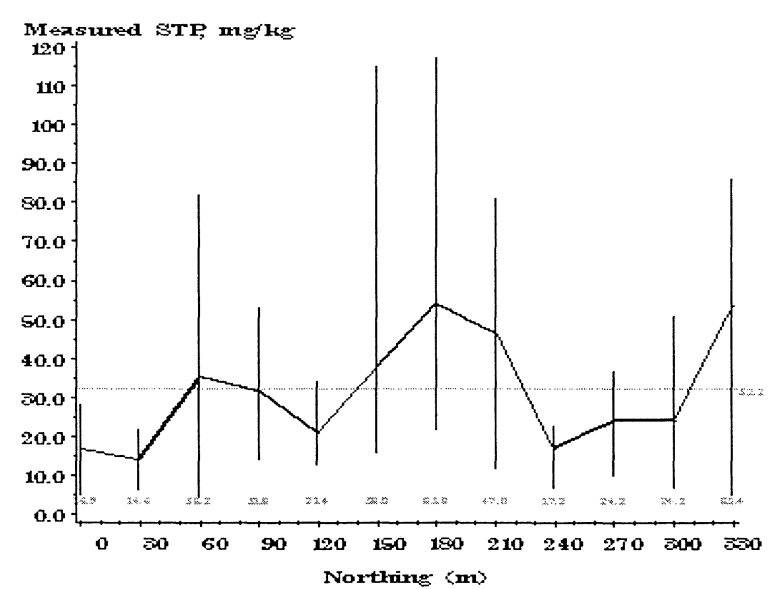




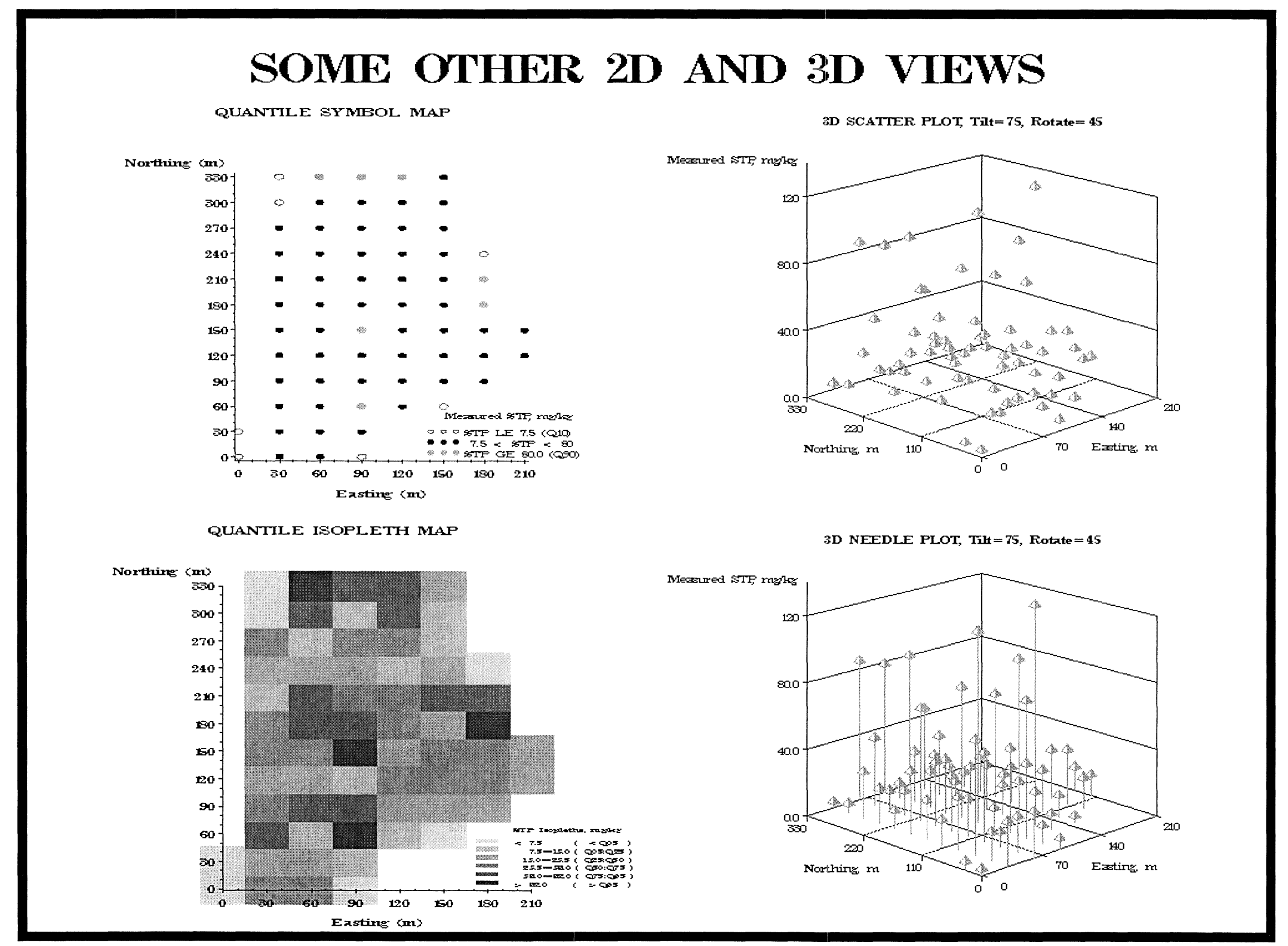




\section{EAST-WEST MODEL VARIOGRAMI}

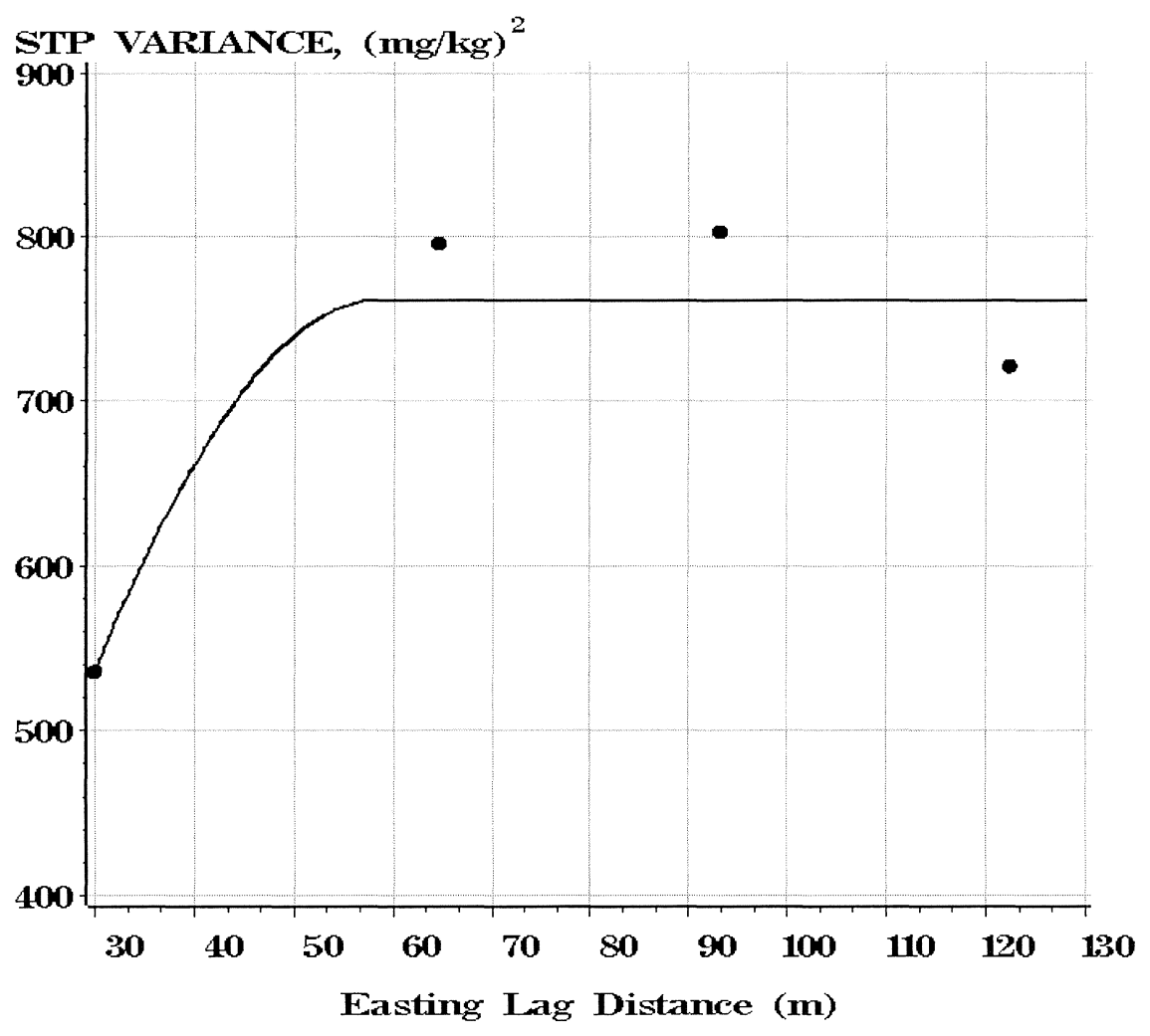

Figure 4. Directional variogram for the stationary STP data (in $\mathrm{mg} / \mathrm{kg}$ ) from Cellar Ridge, AR. 


\section{Data and Kriging Grids for STP ( $\mathrm{mg} / \mathrm{kg}$ )}

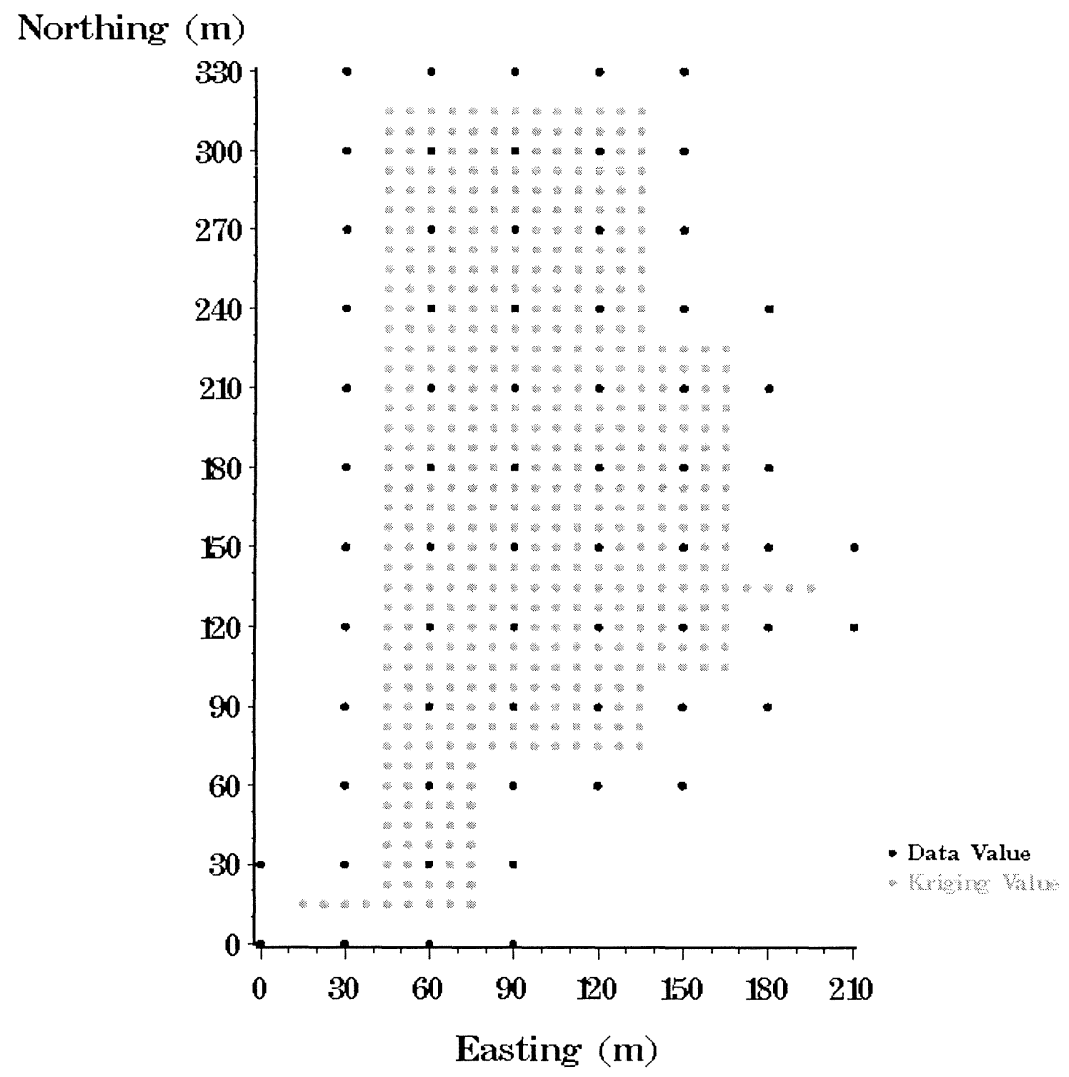

Figure 5. Data and kriging grids for the 1999 STP (mg/kg) study at Cellar Ridge, AR. Note the kriging grid is entirely inside the perimeter of the sampling grid so at least 4 surrounding points can be used for the kriging interpolation procedure. 


\section{CONTOUR/ISOPLETH COMBINATION}

Kriged STP, $\mathrm{mg} / \mathrm{kg}$

Northing (m)

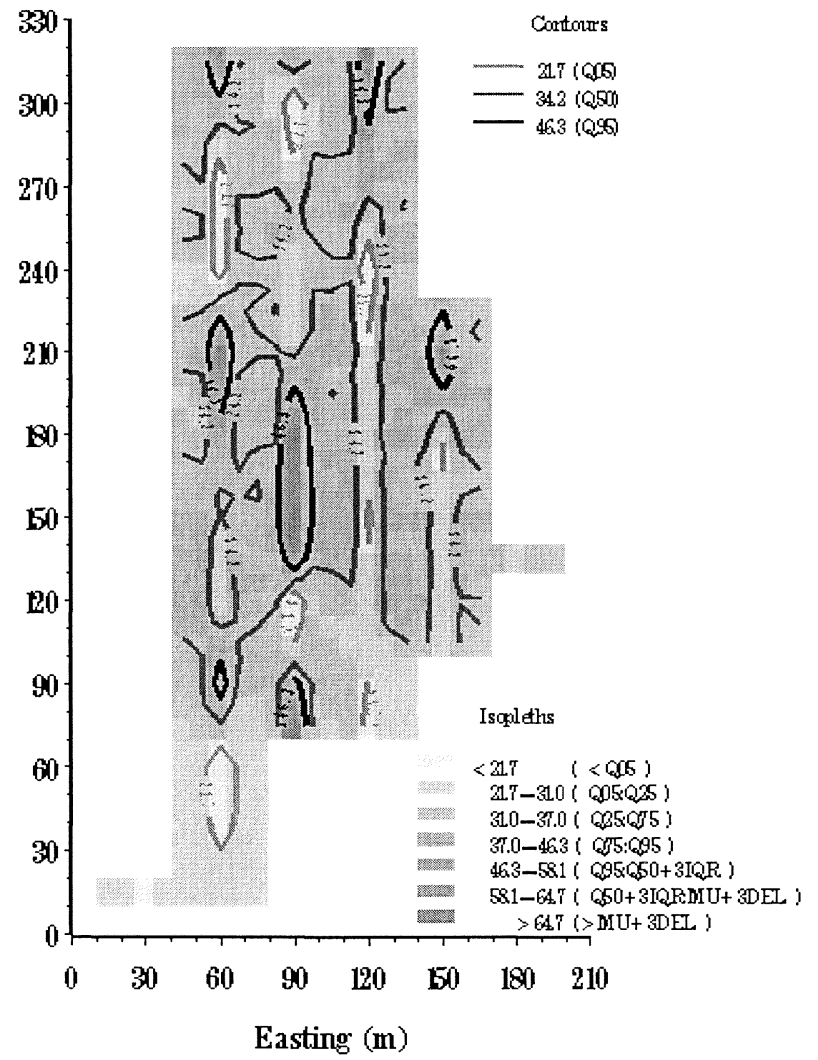

Kriged STP SE, mg/kg

Northing (m)

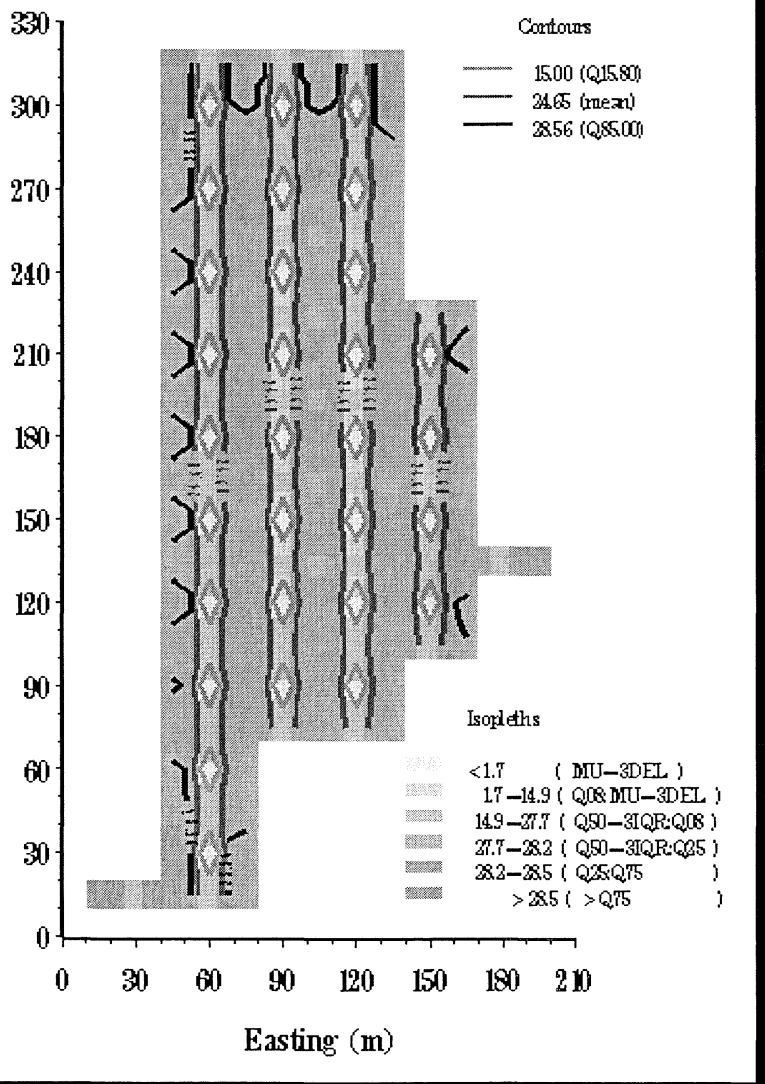

Figure 6. Suggested gray-scale contour/isopleth overlays for Cellar Ridge, AR kriged STP surface and its standard error $(\mathrm{mg} / \mathrm{kg})$ constructed from the 1999 samples.

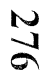

 